\title{
REPRESENTATION THEORY OF SUPERCONFORMAL ALGEBRAS AND THE KAC-ROAN-WAKIMOTO CONJECTURE
}

\author{
TOMOYUKI ARAKAWA
}

\begin{abstract}
We study the representation theory of the superconformal algebra $\mathcal{W}_{k}\left(\mathfrak{g}, f_{\theta}\right)$ associated to a minimal gradation of $\mathfrak{g}$. Here, $\mathfrak{g}$ is a simple finite-dimensional Lie superalgebra with a non-degenerate even supersymmetric invariant bilinear form. Thus, $\mathcal{W}_{k}\left(\mathfrak{g}, f_{\theta}\right)$ can be the Virasoro algebra, the Bershadsky-Polyakov algebra, the Neveu-Schwarz algebra, the BershadskyKnizhnik algebras, the $N=2$ superconformal algebra, the $N=4$ superconformal algebra, the $N=3$ superconformal algebra, the big $N=4$ superconformal algebra, and so on. The conjecture of V. Kac, S.-S. Roan and M. Wakimoto for $\mathcal{W}_{k}\left(\mathfrak{g}, f_{\theta}\right)$ is proved. In fact, we show that any irreducible highest weight character of $\mathcal{W}_{k}\left(\mathfrak{g}, f_{\theta}\right)$ at any level $k \in \mathbb{C}$ is determined by the corresponding irreducible highest weight character of the Kac-Moody affinization of $\mathfrak{g}$.
\end{abstract}

\section{INTRODUCTION}

In this paper we study the representation theory of the vertex algebra $\mathcal{W}_{k}\left(\mathfrak{g}, f_{\theta}\right)$ associated to a minimal gradation of $\mathfrak{g}$, introduced by V. Kac, S.-S. Roan, and M. Wakimoto 10. Here, $\mathfrak{g}$ is a simple finite-dimensional Lie superalgebra with a nondegenerate even supersymmetric invariant bilinear form, $f_{\theta}$ is the lowest root vector of $\mathfrak{g}$ and the level $k \in \mathbb{C}$ is arbitrary. Thus, $\mathcal{W}_{k}\left(\mathfrak{g}, f_{\theta}\right)$ can be the Virasoro algebra, the Bershadsky-Polyakov algebra [3], the Neveu-Schwarz algebra, the BershadskyKnizhnik algebras [4], the $N=2$ superconformal algebra, the $N=4$ superconformal algebra, the $N=3$ superconformal algebra, the big $N=4$ superconformal algebra, and so on, as listed in [10.

Let $\widehat{\mathfrak{g}}$ be the Kac-Moody affinization of $\mathfrak{g}$. In this paper we show that the representation theory of $\mathcal{W}_{k}\left(\mathfrak{g}, f_{\theta}\right)$ is actually controlled by $\widehat{\mathfrak{g}}$ in the following sense.

Let $\mathcal{O}_{k}$ be the Bernstein-Gelfand-Gelfand category of $\widehat{\mathfrak{g}}$ at the level $k$. The construction of [10] gives a family of functors $V \rightsquigarrow H^{i}(V)$ depending on $i \in \mathbb{Z}$, from $\mathcal{O}_{k}$ to the category of $\mathcal{W}_{k}\left(\mathfrak{g}, f_{\theta}\right)$-modules. Here, $H^{\bullet}(V)$ is the BRST cohomology of the corresponding quantum reduction.

Main Theorem 1 (Theorem 8.2.1). We have $H^{i}(V)=\{0\}(i \neq 0)$ for any $V \in \operatorname{Obj}_{k}$.

The above result says in particular that the correspondence $V \rightsquigarrow H^{0}(V)$ defines an exact functor from $\mathcal{O}_{k}$ to the category of $\mathcal{W}_{k}\left(\mathfrak{g}, f_{\theta}\right)$-modules, defining a map between characters.

Main Theorem 2 (Theorem8.3.1). Let $L(\lambda) \in \operatorname{ObjO}_{k}$ be the irreducible $\widehat{\mathfrak{g}}$-module of highest weight $\lambda$. If $\left\langle\lambda, \alpha_{0}^{\vee}\right\rangle \in\{0,1,2, \ldots\}$, then $H^{0}(L(\lambda))=\{0\}$. Otherwise

1991 Mathematics Subject Classification. 17B69, 17B56, 81R10. 
$H^{0}(L(\lambda))$ is isomorphic to the irreducible $\mathcal{W}_{k}\left(\mathfrak{g}, f_{\theta}\right)$-module of the corresponding highest weight.

Every irreducible highest weight module of $\mathcal{W}_{k}\left(\mathfrak{g}, f_{\theta}\right)$ is isomorphic to $H^{0}(L(\lambda))$ for some $\lambda$. Hence, it follows that any irreducible highest weight character of $\mathcal{W}_{k}\left(\mathfrak{g}, f_{\theta}\right)$ is determined by the character of the corresponding irreducible $\widehat{\mathfrak{g}}$-module $L(\lambda)$.

Our results in particular prove the conjecture of V. Kac, S.-S. Roan and M. Wakimoto [10] on the irreduciblity of $H^{0}(L(\lambda))$ for an admissible $\widehat{\mathfrak{g}}$-module $L(\lambda)$. Also, Main Theorem 2 agrees with their computation of the Euler-Poincaré character of $H^{\bullet}(L(\lambda))$.

This paper is organized as follows. In Section 2, we collect the necessary information about the affine Lie superalgebra $\widehat{\mathfrak{g}}$. In Section 3, we recall the definition of the BRST complex constructed by V. Kac, S.-S. Roan, and M. Wakimoto [10]. Although the $\mathcal{W}$-algebra $\mathcal{W}_{k}(\mathfrak{g}, f)$ can be defined for an arbitrary even nilpotent element $f$, the assumption $f=f_{\theta}$ simplifies the theory in many ways. This is also the case when all the interesting superconformal algebras appear, as explained in 10. In section 4, we derive some basic but important facts about the BRST cohomology under the assumption $f=f_{\theta}$. In Section 5 , we recall the definition of $\mathcal{W}$-algebra $\mathcal{W}_{k}(\mathfrak{g}, f)$ and the collect necessary information about its structure. In Section 6 , we recall the parameterization of irreducible highest weight representations of $\mathcal{W}_{k}\left(\mathfrak{g}, f_{\theta}\right)$. We also state the important vanishing theorem (Theorem 6.4.1) which easily follows from a result of V. Kac and M. Wakimoto [1]. The main results in Section 7 are Theorem 7.17.1 and Theorem 7.18.1] To prove them we compute the BRST cohomology $H^{\bullet}\left(M(\lambda)^{*}\right)$ associated to the dual of the Verma module $M(\lambda)$ using a spectral sequence. The argument used here is a modified version of [1]. Finally, in Section 8, we prove Main Theorems.

The method used in this paper can be applied to general $\mathcal{W}$-algebras, with some modifications. The corresponding results will appear in our forthcoming papers.

Acknowledgments. This work was started during my visit to M.I.T. from February to March 2004. I would like to thank the people of M.I.T. for the hospitality, Professor Victor Kac in particular.

\section{Preliminaries}

2.1. Let $\mathfrak{g}$ be a complex simple finite-dimensional Lie superalgebra with a nondegenerate even supersymmetric invariant bilinear form (.|.). Let $(e, x, f)$ be a $\mathfrak{s l}_{2}$-triple in the even part of $\mathfrak{g}$ normalized as follows:

$$
[e, f]=x,[x, e]=e,[x, f]=-f .
$$

Let

$$
\mathfrak{g}=\bigoplus_{j \in \frac{1}{2} \mathbb{Z}} \mathfrak{g}_{j}, \quad \mathfrak{g}_{j}=\{u \in \mathfrak{g} \mid[x, u]=j a\}
$$

be the eigenspace decomposition of $\mathfrak{g}$ with respect to ad $x$.

2.2. Let $\mathfrak{g}^{f}=\{u \in \mathfrak{g} \mid[f, u]=0\}$, the centralizer of $f$ in $\mathfrak{g}$. Then, $\mathfrak{g}^{f}=\sum_{j \leq 0} \mathfrak{g}_{j}^{f}$, where $\mathfrak{g}_{j}^{f}=\mathfrak{g}^{f} \cap \mathfrak{g}_{j}$. Similarly set $\mathfrak{g}^{e}=\sum_{j \geq 0} \mathfrak{g}_{j}^{e}=\{u \in \mathfrak{g} \mid[e, u]=0\}$ and so on. 
2.3. Let

$$
\mathfrak{g}_{\geq 1}=\bigoplus_{j \geq 1} \mathfrak{g}_{j}, \quad \mathfrak{g}_{>0}=\bigoplus_{j>0} \mathfrak{g}_{j}
$$

They are both nilpotent subalgebras of $\mathfrak{g}$. Similarly define $\mathfrak{g}_{\geq 0}, \mathfrak{g}_{<0}, \mathfrak{g}_{\leq-1}$, and so on.

2.4. Define a character $\bar{\chi}$ of $\mathfrak{g}_{\geq 1}$ by

$$
\bar{\chi}(u)=(f \mid u) \quad\left(u \in \mathfrak{g}_{\geq 1}\right) .
$$

Then, it defines a skew-supersymmetric even bilinear form $\langle. \mid \cdot\rangle_{\text {ne }}$ on $\mathfrak{g}_{\frac{1}{2}}$ by the formula

$$
\langle u \mid v\rangle_{\mathrm{ne}}=\bar{\chi}([u, v]) .
$$

Note $\langle. \mid \cdot\rangle_{\text {ne }}$ is non-degenerate by the $\mathfrak{s l}_{2}$-representation theory. We have

$$
\langle u \mid[a, v]\rangle_{\mathrm{ne}}=\langle[u, a] \mid v\rangle_{\mathrm{ne}} \quad\left(a \in \mathfrak{g}_{0}^{f}, u, v \in \mathfrak{g}_{\frac{1}{2}}\right) .
$$

2.5. Let $\mathfrak{h}, x \in \mathfrak{h}$, be a Cartan subalgebra of the even part of $\mathfrak{g}_{0}$. Then, $\mathfrak{h}$ is a Cartan subalgebra of the even part of $\mathfrak{g}$. Let $\Delta_{j} \subset \mathfrak{h}^{*}$ be the set of roots in $\mathfrak{g}_{j}$ and let $\Delta_{0+}$ be a set of positive roots of $\Delta_{0}$. Then, $\Delta=\sqcup_{j \in \frac{1}{2} \mathbb{Z}} \Delta_{j}$ is the set of roots, $\Delta_{+}=\Delta_{0+} \sqcup \Delta_{>0}$ is a set of positive roots of $\mathfrak{g}$, where $\Delta_{>0}=\sqcup_{j>0} \Delta_{j}$. This gives the triangular decompositions

$$
\mathfrak{g}=\mathfrak{n}_{-} \oplus \mathfrak{h} \oplus \mathfrak{n}_{+}, \quad \mathfrak{g}_{0}=\mathfrak{n}_{0,-} \oplus \mathfrak{h} \oplus \mathfrak{n}_{0,+} .
$$

Here, $\mathfrak{n}_{+}=\sum_{\alpha \in \Delta_{+}} \mathfrak{g}_{\alpha}, \mathfrak{n}_{0,+}=\sum_{\alpha \in \Delta_{0,+}} \mathfrak{g}_{\alpha}$, and so on.

2.6. Let $u \mapsto u^{t}$ be an anti-automorphism of $\mathfrak{g}$ such that $e^{t}=f, f^{t}=e, x^{t}=x$, $\mathfrak{g}_{\alpha}^{t}=\mathfrak{g}_{-\alpha}(\alpha \in \Delta)$ and $\left(u^{t} \mid v^{t}\right)=(v \mid u)(u, v \in \mathfrak{g})$.

We fix root vectors $u_{\alpha} \in \mathfrak{g}_{\alpha}, \alpha \in \Delta$, such that $\left(u_{\alpha}, u_{-\alpha}\right)=1$ and $u_{\alpha}^{t}=u_{-\alpha}$ $\left(\alpha \in \Delta_{+}\right)$.

2.7. For $\alpha \in \Delta$, let $p(\alpha)$ be its parity. The parity of $v \in \mathfrak{g}$ is also denote by $p(v)$.

2.8. Let $\widehat{\mathfrak{g}}$ be the Kac-Moody affinization of $\mathfrak{g}$. It is the Lie superalgebra

$$
\widehat{\mathfrak{g}}=\mathfrak{g} \otimes \mathbb{C}\left[t, t^{-1}\right] \oplus \mathbb{C} K \oplus \mathbb{C D}
$$

with the commutation relations

$$
\begin{aligned}
& {[u(m), v(n)]=[u, v](m+n)+m \delta_{m+n, 0}(u \mid v) K,} \\
& {[\mathbf{D}, u(m)]=m u(m), \quad[K, \widehat{\mathfrak{g}}]=0}
\end{aligned}
$$

$(u, v \in \mathfrak{g}, m, n \in \mathbb{Z})$. Here, $u(m)=u \otimes t^{m}, u \in \mathfrak{g}, m \in \mathbb{Z}$.

The invariant bilinear form $(\cdot \mid \cdot)$ is extended from $\mathfrak{g}$ to $\widehat{\mathfrak{g}}$ by letting $(u(m) \mid v(n))=$ $(u \mid v) \delta_{m+n, 0}(u, v \in \mathfrak{g}, m, n \in \mathbb{Z}),\left(\mathfrak{g} \otimes \mathbb{C}\left[t, t^{-1}\right], \mathbb{C} K \oplus \mathbb{C D}\right)=0,(K, K)=(\mathbf{D}, \mathbf{D})=$ $0,(K, \mathbf{D})=(\mathbf{D}, K)=1$.

2.9. Define subalgebras

$$
L \mathfrak{g}_{\geq 1}=\mathfrak{g}_{\geq 1} \otimes \mathbb{C}\left[t, t^{-1}\right], \quad L \mathfrak{g}_{>0}=\mathfrak{g}_{>0} \otimes \mathbb{C}\left[t, t^{-1}\right] \subset \widehat{\mathfrak{g}} .
$$

Similarly define $L \mathfrak{g}_{\geq 0}, L \mathfrak{g}_{<0}, L \mathfrak{g}_{\leq-1}$, and so on. 
2.10. Fix the triangular decomposition $\widehat{\mathfrak{g}}=\widehat{\mathfrak{n}}_{-} \oplus \widehat{\mathfrak{h}} \oplus \widehat{\mathfrak{n}}_{+}$in the standard way. Thus,

$$
\begin{aligned}
& \widehat{\mathfrak{h}}=\mathfrak{h} \oplus \mathbb{C} K \oplus \mathbb{C D}, \\
& \widehat{\mathfrak{n}}_{-}=\mathfrak{n}_{-} \otimes \mathbb{C}\left[t^{-1}\right] \oplus \mathfrak{h} \otimes \mathbb{C}\left[t^{-1}\right] t^{-1} \oplus \mathfrak{n}_{+} \otimes \mathbb{C}\left[t^{-1}\right] t^{-1}, \\
& \widehat{\mathfrak{n}}_{+}=\mathfrak{n}_{-} \otimes \mathbb{C}[t] t \oplus \mathfrak{h} \otimes \mathbb{C}[t] t \oplus \mathfrak{n}_{+} \otimes \mathbb{C}[t] .
\end{aligned}
$$

Let $\widehat{\mathfrak{h}}^{*}=\mathfrak{h}^{*} \oplus \mathbb{C} \Lambda_{0} \oplus \mathbb{C} \delta$ be the dual of $\widehat{\mathfrak{h}}$. Here, $\Lambda_{0}$ and $\delta$ are dual elements of $K$ and $\mathbf{D}$ respectively.

Let $\widehat{\Delta}$ be the set of roots of $\widehat{\mathfrak{g}}, \widehat{\Delta}_{+}$the set of positive roots, and $\widehat{\Delta}_{-}=-\widehat{\Delta}_{+}$. Let $\widehat{Q}$ be the root lattice and $\widehat{Q}_{+}=\sum_{\alpha \in \widehat{\Delta}_{+}} \mathbb{Z}_{\geq 0} \alpha \subset \widehat{Q}$. We define a partial ordering $\mu \leq \lambda$ on $\widehat{\mathfrak{h}}^{*}$ by $\lambda-\mu \in \widehat{Q}_{+}$.

2.11. For an $\widehat{\mathfrak{h}}$-module $V$, let $V^{\lambda}=\{v \in V \mid h v=\lambda(h) v$ for all $h \in \widehat{\mathfrak{h}}\}$. If all the weight spaces $V^{\lambda}$ are finite-dimensional, we set

$$
V^{*}=\bigoplus_{\lambda \in \mathfrak{h}^{*}} \operatorname{Hom}_{\mathbb{C}}\left(V^{\lambda}, \mathbb{C}\right) \subset \operatorname{Hom}_{\mathbb{C}}(V, \mathbb{C}) .
$$

2.12. For $k \in \mathbb{C}$, let $\widehat{\mathfrak{h}}_{k}^{*}$ denote the set of weights of level $k$ :

$$
\widehat{\mathfrak{h}}_{k}^{*}=\left\{\lambda \in \widehat{\mathfrak{h}}^{*} \mid\langle\lambda, K\rangle=k\right\} .
$$

Let $\mathcal{O}_{k}$ be the full subcategory of the category of left $\widehat{\mathfrak{g}}$-modules consisting of objects $V$ such that

(1) $V=\bigoplus_{\lambda \in \widehat{\mathfrak{h}}_{k}^{*}} V^{\lambda}$ and $\operatorname{dim}_{\mathbb{C}} V^{\lambda}<\infty$ for all $\lambda \in \widehat{\mathfrak{h}}_{k}^{*}$,

(2) there exists a finite set $\left\{\mu_{1}, \ldots, \mu_{r}\right\} \subset \widehat{\mathfrak{h}}_{k}^{*}$ such that $\lambda \in \bigcup_{i}\left(\mu_{i}-\widehat{Q}_{+}\right)$for any $\lambda$ with $V^{\lambda} \neq\{0\}$.

Then, $\mathcal{O}_{k}$ is an abelian category. Let $M(\lambda) \in \operatorname{Obj}_{k}, \lambda \in \widehat{\mathfrak{h}}_{k}^{*}$, be the Verma module of highest weight $\lambda$. Thus, $M(\lambda)=U(\widehat{\mathfrak{g}}) \otimes_{U\left(\widehat{\mathfrak{h}} \oplus \widehat{\mathfrak{n}}_{+}\right)} \mathbb{C}_{\lambda}$, where $\mathbb{C}_{\lambda}$ is the onedimensional $\widehat{\mathfrak{h}} \oplus \widehat{\mathfrak{n}}_{+}$-module on which $\widehat{\mathfrak{n}}_{+}$acts trivially and $h \in \widehat{\mathfrak{h}}$ acts as $\langle\lambda, h\rangle$ id. Let $v_{\lambda}$ be the highest weight vector of $M(\lambda)$. Let $L(\lambda) \in \operatorname{Obj} \mathcal{O}_{k}$ be the unique simple quotient of $M(\lambda)$.

2.13. The correspondence $V \rightsquigarrow V^{*}$ defines the duality functor in $\mathcal{O}_{k}$. Here, $\widehat{\mathfrak{g}}$ acts on $V^{*}$ by $(a f)(v)=f\left(a^{t} v\right)$, where $a \mapsto a^{t}$ is the antiautomorphism of $\widehat{\mathfrak{g}}$ defined by $u(m)^{t}=\left(u^{t}\right)(-m)(u \in \mathfrak{g}, m \in \mathbb{Z}), K^{t}=K, \mathbf{D}^{t}=\mathbf{D}$. We have: $L(\lambda)^{*}=L(\lambda)$ $\left(\forall \lambda \in \widehat{\mathfrak{h}}_{k}^{*}\right)$.

2.14. Let $\mathcal{O}_{k}^{\triangle}$ be the full subcategory of $\mathcal{O}_{k}$ consisting of objects $V$ that admits a Verma flag, that is, a finite filtration $V=V_{0} \supset V_{1} \supset \cdots \supset V_{r}=\{0\}$ such that each successive subquotient $V_{i} / V_{i+1}$ is isomorphic to some Verma module $M(\lambda)$. The category $\mathcal{O}_{k}^{\triangle}$ is stable under taking direct summands. Dually, let $\mathcal{O}_{k}^{\nabla}$ be the full subcategory of $\mathcal{O}_{k}$ consisting of objects $V$ such that $V^{*} \in \operatorname{Obj}_{k}^{\triangle}$. 
2.15. For $\lambda \in \widehat{\mathfrak{h}}_{k}^{*}$, let $\mathcal{O}_{\bar{k}}^{\leq \lambda}$ be the full subcategory of $\mathcal{O}_{k}$ consisting of objects $V$ such that $V=\bigoplus_{\mu \leq \lambda} V^{\mu}$. Then, $\mathcal{O}_{k}^{\leq \lambda}$ is an abelian category and stable under taking duals. Also, every simple object $L(\mu) \in \mathcal{O}_{k}^{\leq \lambda}$ admits a projective cover $P_{\leq \lambda}(\mu)$ in $\mathcal{O}_{k}^{\leq \lambda}$, and hence, every finitely generated object in $\mathcal{O}_{k}^{\leq \lambda}$ is an image of some projective object. Indeed, as in the Lie algebra case, $P_{\leq \lambda}(\mu)$ can be defined as an indecomposable direct summand of

$$
U(\widehat{\mathfrak{g}}) \otimes_{U\left(\widehat{\mathfrak{h}} \oplus \widehat{\mathfrak{g}}_{+}\right)} \tau_{\leq \lambda}\left(U\left(\widehat{\mathfrak{h}} \oplus \widehat{\mathfrak{n}}_{+}\right) \otimes_{U(\widehat{\mathfrak{h}})} \mathbb{C}_{\mu}\right)
$$

which has $L(\mu)$ as a quotient. Here, $\tau_{\leq \lambda}(V)=V / \bigoplus_{\substack{\nu \in \hat{h}^{*} \\ \nu \not \lambda \lambda}} V^{\nu}$, and $\mathbb{C}_{\mu}$ is a one-

dimensional $\widehat{\mathfrak{h}}$-module on which $h \in \widehat{\mathfrak{h}}$ acts as $\mu(h)$ id. Note that $P_{\leq \lambda}(\mu) \in \operatorname{Obj} \mathcal{O}_{k}^{\triangle}$. Moreover, the BGG(Bernstein-Gelfand-Gelfand) reciprocity holds:

$$
\left[P_{\leq \lambda}(\mu): M\left(\mu^{\prime}\right)\right]=\left[M\left(\mu^{\prime}\right): L(\mu)\right] \quad\left(\mu, \mu^{\prime} \leq \lambda\right) .
$$

Here, $\left[P_{\leq \lambda}(\mu): M\left(\mu^{\prime}\right)\right]$ is the multiplicity of $M\left(\mu^{\prime}\right)$ in the Verma flag of $P_{\leq \lambda}(\mu)$, and $\left[M\left(\mu^{\prime}\right): L(\mu)\right]$ is the multiplicity of $L(\mu)$ in the local composition factor of $M\left(\mu^{\prime}\right)$. Dually, $I_{\leq \lambda}(\mu)=P_{\leq \lambda}(\mu)^{*}$ is the injective envelope of $L(\mu)$ in $\mathcal{O}_{k}^{\leq \lambda}$. In particular, $V \in \operatorname{Obj} \mathcal{O}_{k}^{\leq \lambda}$ is a submodule of some injective object if its dual $V^{*}$ is finitely generated.

\section{KaC-RoAn-Wakimoto CONSTRUCTION I: the BRST COMPLEX}

3.1. Define a character $\chi$ of $L \mathfrak{g}_{\geq 1}$ by

$$
\chi(u(m))=(f(1) \mid u(m))=\bar{\chi}(u) \delta_{m,-1} \quad\left(u \in \mathfrak{g}_{\geq 1}, m \in \mathbb{Z}\right) .
$$

Let ker $\chi \subset U\left(L \mathfrak{g}_{\geq 1}\right)$ be the kernel of the algebra homomorphism $\chi: U\left(L \mathfrak{g}_{\geq 1}\right) \rightarrow \mathbb{C}$. Set $I_{\chi}=U\left(L \mathfrak{g}_{>0}\right)$ ker $\chi$. Then, $I_{\chi}$ is a two sided ideal of $U\left(L \mathfrak{g}_{>0}\right)$. Define

$$
N(\chi)=U\left(L \mathfrak{g}_{>0}\right) / I_{\chi} .
$$

Let $\Phi_{u}(n)\left(u \in \mathfrak{g}_{>0}, n \in \mathbb{Z}\right)$ denote the image of $u(n) \in L \mathfrak{g}_{>0}$ in $N(\chi)$. Abusing notation, we set $\Phi_{\alpha}(n)=\Phi_{u_{\alpha}}(n)$ for $\alpha \in \Delta_{\frac{1}{2}}$ and $n \in \mathbb{Z}$. Then, the superalgebra $N(\chi)$ is generated by $\Phi_{\alpha}(n), \alpha \in \Delta_{\frac{1}{2}}, n \in \mathbb{Z}$, with the relations

$$
\left[\Phi_{\alpha}(m), \Phi_{\beta}(n)\right]=\left\langle u_{\alpha} \mid u_{\beta}\right\rangle_{\mathrm{ne}} \delta_{m+n,-1} \quad\left(\alpha, \beta \in \Delta_{\frac{1}{2}}, m, n \in \mathbb{Z}\right) .
$$

Let $\left\{u^{\alpha}\right\}_{\alpha \in \Delta_{\frac{1}{2}}}$ be the basis of $\mathfrak{g}_{\frac{1}{2}}$ dual to $\left\{u_{\alpha}\right\}_{\alpha \in \Delta_{\frac{1}{2}}}$ with respect to $\langle\mid\rangle_{\text {ne }}$, that is, $\left\langle u_{\alpha} \mid u^{\beta}\right\rangle_{\mathrm{ne}}=\delta_{\alpha, \beta}$. We set $\Phi^{\alpha}(n)=\Phi_{u^{\alpha}}(n)\left(\alpha \in \Delta_{\frac{1}{2}}, n \in \mathbb{Z}\right)$, so

$$
\left[\Phi_{\alpha}(m), \Phi^{\beta}(n)\right]=\delta_{\alpha, \beta} \delta_{m+n,-1} .
$$

The elements $\left\{\Phi_{\alpha}(n)\right\}$ are called the Neutral free superfermions.

3.2. Let $\mathcal{F}^{\text {ne }}(\chi)$ be the irreducible representations of $N(\chi)$ generated by a vector $\mathbf{1}_{\chi}$ such that

$$
\Phi_{\alpha}(n) \mathbf{1}_{\chi}=0 \quad\left(\alpha \in \Delta_{\frac{1}{2}}, n \geq 0\right) .
$$

The space $\mathcal{F}^{\text {ne }}(\chi)$ is naturally a $L \mathfrak{g}_{>0}$-module via the algebra homomorphism $L \mathfrak{g}_{>0} \ni u(m) \mapsto \Phi_{u}(m) \in N(\chi)$. 
There is a unique semisimple action of $\widehat{\mathfrak{h}}$ on $\mathcal{F}^{\mathrm{ne}}(\chi)$ such that

$$
\begin{aligned}
& h \mathbf{1}_{\chi}=0 \quad(h \in \mathfrak{h}), \\
& \Phi_{\alpha}(n) \mathcal{F}^{\mathrm{ne}}(\chi)^{\lambda} \subset \mathcal{F}^{\mathrm{ne}}(\chi)^{\lambda+\alpha+n \delta}\left(\alpha \in \Delta_{\frac{1}{2}}, n \leq-1, \lambda \in \widehat{\mathfrak{h}}^{*}\right) .
\end{aligned}
$$

Note by this definition we have

$$
\Phi^{\alpha}(n) \mathcal{F}^{\mathrm{ne}}(\chi)^{\lambda} \subset \mathcal{F}^{\mathrm{ne}}(\chi)^{\lambda-\alpha+(n+1) \delta} \text { for } n \geq 0,
$$

see (16).

3.3. Let $\mathcal{C l}\left(L \mathfrak{g}_{>0}\right)$ be the Clifford superalgebra, or the Charged free superfermions, associated to $L \mathfrak{g}_{>0} \oplus\left(L \mathfrak{g}_{>0}\right)^{*}$ and its natural bilinear from. The superalgebra $\mathcal{C l}\left(L \mathfrak{g}_{>0}\right)$ is generated by $\psi_{\alpha}(n), \psi^{\alpha}(n)\left(\alpha \in \Delta_{>0}, n \in \mathbb{Z}\right)$ with the relations

$$
\begin{aligned}
& {\left[\psi_{\alpha}(m), \psi^{\beta}(n)\right]=\delta_{\alpha, \beta} \delta_{m+n, 0},} \\
& {\left[\psi_{\alpha}(m), \psi_{\beta}(m)\right]=\left[\psi^{\alpha}(m), \psi^{\beta}(n)\right]=0,}
\end{aligned}
$$

where the parity of $\psi_{\alpha}(n)$ and $\psi^{\alpha}(n)$ are reverse to $u_{\alpha}$.

3.4. Let $\mathcal{F}\left(L \mathfrak{g}_{>0}\right)$ be the irreducible representation of $\mathcal{C l}\left(L \mathfrak{g}_{>0}\right)$ generated by the vector 1 such that

$$
\psi_{\alpha}(n) \mathbf{1}=0 \quad\left(\alpha \in \Delta_{>0}, n \geq 0\right), \quad \psi^{\alpha}(n) \mathbf{1}=0 \quad\left(\alpha \in \Delta_{>0}, n>0\right) .
$$

The space $\mathcal{F}\left(L \mathfrak{g}_{>0}\right)$ is graded: $\mathcal{F}\left(L \mathfrak{g}_{>0}\right)=\bigoplus_{i \in \mathbb{Z}} \mathcal{F}^{i}\left(L \mathfrak{g}_{>0}\right)$, where the degree is counted from $\operatorname{deg} \mathbf{1}=0, \operatorname{deg} \psi_{\alpha}(n)=-1, \operatorname{deg} \psi^{\alpha}(n)=1\left(\alpha \in \Delta_{>0}, n \in \mathbb{Z}\right)$.

There is a natural semisimple $\widehat{\mathfrak{h}}$-action on $\mathcal{F}\left(L \mathfrak{g}_{>0}\right): \mathcal{F}\left(L \mathfrak{g}_{>0}\right)=\bigoplus_{\lambda \in \widehat{\mathfrak{h}}^{*}} \mathcal{F}\left(L \mathfrak{g}_{>0}\right)^{\lambda}$. It is defined by $h \mathbf{1}=0(h \in \widehat{\mathfrak{h}}), \psi_{\alpha}(n) \mathcal{F}\left(L \mathfrak{g}_{>0}\right)^{\lambda} \subset \mathcal{F}\left(L \mathfrak{g}_{>0}\right)^{\lambda+\alpha+n \delta}, \psi^{\alpha}(n) \mathcal{F}\left(L \mathfrak{g}_{>0}\right)^{\lambda} \subset$ $\mathcal{F}\left(L \mathfrak{g}_{>0}\right)^{\lambda-\alpha+n \delta}\left(\alpha \in \Delta_{>0}, n \in \mathbb{Z}\right)$.

3.5. For $V \in \operatorname{Obj}_{k}$, let

$$
C(V)=V \otimes \mathcal{F}^{\mathrm{ne}}(\chi) \otimes \mathcal{F}\left(L \mathfrak{g}_{>0}\right)=\sum_{i \in \mathbb{Z}} C^{i}\left(L \mathfrak{g}_{>0}, V\right),
$$

where $C^{i}(V)=V \otimes \mathcal{F}^{\mathrm{ne}}(\chi) \otimes \mathcal{F}^{i}\left(L \mathfrak{g}_{>0}\right)$.

Let $\widehat{\mathfrak{h}}$ act on $C(V)$ by the tensor product action. Then, $C(V)=\bigoplus_{\lambda \in \widehat{\mathfrak{h}}^{*}} C(V)^{\lambda}$, $C(V)^{\lambda}=\sum_{\mu_{1}+\mu_{2}+\mu_{3}=\lambda} V^{\mu_{1}} \otimes \mathcal{F}^{\text {ne }}(\chi)^{\mu_{2}} \otimes \mathcal{F}\left(L \mathfrak{g}_{>0}\right)^{\mu_{3}}$. Note that

$$
C(V)=\bigoplus_{\mu \leq \lambda} C(V)^{\mu}, \quad \operatorname{dim}_{\mathbb{C}} C(V)^{\mu}<\infty(\forall \mu)
$$

for $V \in \operatorname{ObjO}_{k}^{\leq \lambda}$.

3.6. Define the odd operator $d$ on $C(V)$ by

$$
\begin{aligned}
d=\sum_{\substack{\alpha \in \Delta>0 \\
n \in \mathbb{Z}}}(-1)^{p(\alpha)}\left(u_{\alpha}(-n)+\Phi_{u_{\alpha}}(-n)\right) \psi^{\alpha}(n) & \\
& -\frac{1}{2} \sum_{\substack{\alpha, \beta, \gamma \in \Delta_{>}>0 \\
k+l+m=0}}(-1)^{p(\alpha) p(\gamma)}\left(\left[u_{\alpha}, u_{\beta}\right] \mid u_{-\gamma}\right) \psi^{\alpha}(k) \psi^{\beta}(l) \psi_{\gamma}(m) .
\end{aligned}
$$

Then, we have

$$
d^{2}=0, \quad d C^{i}(V) \subset C^{i+1}(V) .
$$


We now define

$$
H^{i}(V)=H^{i}(C(V), d) \quad(i \in \mathbb{Z}) .
$$

Remark 3.6.1. By the definition,

$$
H^{\bullet}(V)=H^{\frac{\infty}{2}+\bullet}\left(L \mathfrak{g}_{>0}, V \otimes \mathcal{F}^{\text {ne }}(\chi)\right),
$$

where $H^{\frac{\infty}{2}+} \cdot\left(L \mathfrak{g}_{>0}, V\right)$ is the semi-infinite cohomology of the Lie superalgebra $L \mathfrak{g}_{>0}$ with coefficients in $V$.

3.7. Decompose $d$ as $d=d^{\chi}+d^{\text {st }}$, where

$$
d^{\chi}=\sum_{\substack{\alpha \in \Delta_{\frac{1}{2}} \\ n \geq 0}}(-1)^{p(\alpha)} \Phi_{\alpha}(n) \psi^{\alpha}(-n)+\sum_{\alpha \in \Delta_{1}}(-1)^{p(\alpha)} \chi\left(u_{\alpha}(-1)\right) \psi^{\alpha}(1)
$$

and $d^{\text {st }}=d-d^{\chi}$. Then,

$$
d^{\chi} C(V)^{\lambda} \subset \sum_{\substack{\alpha \in \Delta_{1} \\ \bar{\chi}\left(u_{\alpha}\right) \neq 0}} C(V)^{\lambda-\alpha+\delta}, \quad d^{\mathrm{st}} C(V)^{\lambda} \subset C(V)^{\lambda}
$$

for all $\lambda$, see (18). Therefore, by (22), it follows that

$$
\left(d^{\chi}\right)^{2}=\left(d^{\text {st }}\right)^{2}=\left\{d^{\chi}, d^{\text {st }}\right\}=0 .
$$

Remark 3.7.1. We have

$$
H^{\bullet}\left(C(V), d^{\text {st }}\right)=H^{\frac{\infty}{2}+\bullet}\left(L \mathfrak{g}_{>0}, V \otimes \mathcal{F}^{\mathrm{ne}}\left(\chi_{0}\right)\right),
$$

where $\mathcal{F}^{\text {ne }}\left(\chi_{0}\right)$ is the $L \mathfrak{g}_{>0}$-module associated to the trivial character $\chi_{0}$ of $L \mathfrak{g}_{\geq 1}$ similarly defined as $\mathcal{F}^{\text {ne }}(\chi)$.

3.8. Define

$$
\mathbf{D}^{\mathcal{W}}=x+\mathbf{D} \in \widehat{\mathfrak{h}}
$$

and set

$$
\widehat{\mathfrak{t}}=\mathfrak{h}^{f} \oplus \mathbb{C} \mathbf{D}^{\mathcal{W}} \subset \widehat{\mathfrak{h}} .
$$

Let $\widehat{\mathfrak{t}}^{*}$ be the dual of $\widehat{\mathfrak{t}}$. For $\lambda \in \widehat{\mathfrak{h}}^{*}$, let $\xi_{\lambda} \in \widehat{\mathfrak{t}}^{*}$ denote its restriction to $\widehat{\mathfrak{t}}$. Let

$$
C(V)=\bigoplus_{\xi \in \widehat{\mathfrak{t}}^{*}} C(V)_{\xi}, \quad C(V)_{\xi}=\sum_{\substack{\lambda \in \hat{\mathfrak{h}}^{*} \\ \xi_{\lambda}=\xi}} C(V)^{\lambda},
$$

be the weight space decomposition with respect to the action of $\widehat{\mathfrak{t}} \subset \widehat{\mathfrak{h}}$. Here and throughout, $M_{\xi}=\{m \in M \mid t m=\langle\xi, t\rangle m(\forall t \in \hat{\mathfrak{t}})\}$ for a $\mathfrak{t}$-module $M$.

By (25), we see that

$$
d C(V)_{\xi} \subset C(V)_{\xi}, V \in \operatorname{Obj}_{\mathcal{O}} \text {, for any } \xi .
$$

Hence the cohomology space $H^{\bullet}(V)$ decomposes as

$$
H^{\bullet}(V)=\bigoplus_{\xi \in \widehat{\mathfrak{t}}^{*}} H^{\bullet}(V)_{\xi}, \quad H^{\bullet}(V)_{\xi}=H^{\bullet}\left(C(V)_{\xi}, d\right)
$$

Notice that the weight space $C(V)_{\xi}, \xi \in \widehat{\mathfrak{t}}^{*}$, is not finite-dimensional in general for $\widehat{\mathfrak{t}}, e(-1)]=0$.

Remark 3.8.1. As we will see in Remark 5.3.1 the operator $\mathbf{D}^{\mathcal{W}}$ is essentially the minus of the zero-mode $L(0)$ of the Virasoro field. 


\section{The assumption $f=f_{\theta}$}

4.1. The gradation (2) is called minimal if

$$
\mathfrak{g}=\mathfrak{g}_{-1} \oplus \mathfrak{g}_{-\frac{1}{2}} \oplus \mathfrak{g}_{0} \oplus \mathfrak{g}_{\frac{1}{2}} \oplus \mathfrak{g}_{1}, \quad \mathfrak{g}_{-1}=\mathbb{C} f \text { and } \quad \mathfrak{g}_{1}=\mathbb{C} e .
$$

As shown in 11, in this case one can choose a root system of $\mathfrak{g}$ so that $e=e_{\theta}$ and $f=f_{\theta}$, the roots vectors attached to $\theta$ and $-\theta$, where $\theta$ is the corresponding highest root.

The condition (32) simplifies the theory in many ways. We assume that $f=f_{\theta}$ and the condition (32) is satisfied until the next section. Also, we normalize ( | ) as $(\theta \mid \theta)=2$.

4.2. We have:

$$
\begin{aligned}
\mathfrak{g}^{f} & =\mathfrak{g}_{-1} \oplus \mathfrak{g}_{-\frac{1}{2}} \oplus \mathfrak{g}_{0}^{f}, \\
\mathfrak{g}_{0}^{f} & =\mathfrak{n}_{0,-} \oplus \mathfrak{h}^{f} \oplus \mathfrak{n}_{0,+}
\end{aligned}
$$

by the $\mathfrak{s l}_{2}$-representation theory. In particular,

$$
\mathfrak{h}=\mathfrak{h}^{f} \oplus \mathbb{C} x, \quad \mathfrak{n}_{-} \subset \mathfrak{g}^{f},
$$

and we have the exact sequence

$$
\begin{aligned}
0 \rightarrow \mathbb{C} \alpha_{0} \oplus \mathbb{C} \Lambda_{0} \hookrightarrow \widehat{\mathfrak{h}}^{*} & \rightarrow \widehat{\mathfrak{t}}^{*} \rightarrow 0 \\
\lambda & \mapsto \xi_{\lambda} .
\end{aligned}
$$

Here, $\alpha_{0}=\delta-\theta$.

4.3. Let $\widehat{\mathfrak{g}}=\bigoplus_{\eta \in \hat{\mathfrak{t}}^{*}}(\widehat{\mathfrak{g}})_{\eta}$ be the weight space decomposition with respect to the adjoint action of $\widehat{\mathfrak{t}}$. We have

$$
(\widehat{\mathfrak{g}})_{0}=\widehat{\mathfrak{h}} \oplus \mathbb{C} e(-1) \oplus \mathbb{C} f(1)
$$

(recall $e=e_{\theta}$ and $f=f_{\theta}$ ). Define

$$
\begin{aligned}
& \widehat{\Delta}^{\mathfrak{t}}=\left\{\eta \in \widehat{\mathfrak{t}}^{*} \mid \eta \neq 0,(\widehat{\mathfrak{g}})_{\eta} \neq\{0\}\right\} \subset \widehat{\mathfrak{t}}^{*} . \\
& \widehat{\Delta}_{+}^{\mathfrak{t}}=\left\{\eta \in \widehat{\Delta}^{\mathfrak{t}} \mid(\widehat{\mathfrak{g}})_{\eta} \subset \widehat{\mathfrak{n}}_{+}\right\} .
\end{aligned}
$$

Then,

$$
\left\langle\eta, \mathbf{D}^{\mathcal{W}}\right\rangle \geq 0 \quad\left(\eta \in \widehat{\Delta}_{+}^{\mathfrak{t}}\right)
$$

Let

$$
\widehat{Q}_{+}^{\mathfrak{t}}=\sum_{\eta \in \widehat{\Delta}_{+}^{\mathfrak{t}}} \mathbb{Z}_{\geq 0} \eta \subset \widehat{\mathfrak{t}}^{*}
$$

and define a partial ordering on $\widehat{\mathfrak{t}}^{*}$ by $\xi \leq \xi^{\prime} \Longleftrightarrow \xi^{\prime}-\xi \in \widehat{Q}_{+}^{\mathfrak{t}}$. Note that the map $\widehat{\mathfrak{h}}^{*} \ni \lambda \mapsto \xi_{\lambda} \in \widehat{\mathfrak{t}}^{*}$ is a homomorphism of partially ordered sets, i.e, $\xi_{\mu} \leq \xi_{\lambda}$ if $\mu \leq \lambda$. 
4.4. We have:

$$
\begin{aligned}
& \mathcal{F}^{\mathrm{ne}}(\chi)=\bigoplus_{\xi \leq 0} \mathcal{F}^{\mathrm{ne}}(\chi)_{\xi}, \quad \operatorname{dim}_{\mathbb{C}} \mathcal{F}^{\mathrm{ne}}(\chi)_{\xi}<\infty(\forall \xi), \quad \mathcal{F}^{\mathrm{ne}}(\chi)_{0}=\mathbb{C} \mathbf{1}_{\chi}, \\
& \mathcal{F}\left(L \mathfrak{g}_{>0}\right)=\bigoplus_{\xi \leq 0} \mathcal{F}\left(L \mathfrak{g}_{>0}\right)_{\xi}, \quad \mathcal{F}\left(L\left(\mathfrak{g}_{>0}\right)\right)_{0}=\mathbb{C} \mathbf{1} \oplus \mathbb{C} \psi_{\theta}(-1) \mathbf{1} .
\end{aligned}
$$

Moreover, for a given $\lambda \in \widehat{\mathfrak{h}}^{*}$,

$$
V=\bigoplus_{\xi \leq \xi_{\lambda}} V_{\xi} \quad\left(V \in \mathcal{O}_{k}^{\leq \lambda}\right)
$$

Hence we have

$$
H^{\bullet}(V)=\bigoplus_{\xi \leq \xi_{\lambda}} H^{\bullet}(V)_{\xi} \quad\left(V \in \mathcal{O}_{k}^{\leq \lambda}\right)
$$

4.5. Here and throughout, we identify $\mathcal{F}\left(L\left(\mathfrak{g}_{>0}\right)\right)_{0}$ with the exterior power module $\Lambda(\mathbb{C} e(-1))$, see (42). Let $\mathbb{C}_{\chi}$ be the one-dimensional module over the commutative Lie algebra $\mathbb{C} e(-1)$ defined by the character $\chi_{\mid \mathbb{C} e(-1)}$. Then, for $V \in \operatorname{Obj}_{\vec{k}}^{\leq \lambda}$, the subcomplex $\left(C(V)_{\xi_{\lambda}}, d\right)$ is nothing but the Chevalley complex for calculating the usual Lie algebra homology $H_{\bullet}\left(\mathbb{C} e(-1), V_{\xi_{\lambda}} \otimes \mathbb{C}_{\chi}\right)$ (with the opposite grading):

Lemma 4.5.1. Let $V \in \operatorname{Obj}_{\widehat{k}_{k}}^{\leq \lambda}, \lambda \in \widehat{\mathfrak{h}}_{k}^{*}$. Then,

$$
H^{i}(V)_{\xi_{\lambda}}= \begin{cases}H_{-i}\left(\mathbb{C} e(-1), V_{\xi_{\lambda}} \otimes \mathbb{C}_{\chi}\right) & (i=0,-1) \\ \{0\} & (\text { otherwise }) .\end{cases}
$$

4.6. Let $\mathcal{O}\left(\mathfrak{s l}_{2}\right)$ be the BGG category of $\mathfrak{s l}_{2}=\langle e, x, f\rangle$, see (11). That is, the full subcategory of the category of left $\mathfrak{s l}_{2}$-modules consisting of modules $V$ such that (1) $V$ is finitely generated over $\mathfrak{s l}_{2},(2) e$ acts locally nilpotently on $V$, (3) $x$ acts semisimply on $V$ and each weight space is finite-dimensional. Let $\bar{M}_{\mathfrak{s l}_{2}}(a) \in$ $\operatorname{ObjO}\left(\mathfrak{s l}_{2}\right)$ be the Verma module of highest weight $a \in \mathbb{C}$, and $\bar{L}_{\mathfrak{s l}_{2}}(a)$ its unique simple quotient. Here, the highest weight is the largest eigenvalue of $2 x$.

Let $\mathbb{C}_{\bar{\chi}_{-}}$be the one-dimensional $\mathbb{C} f$-module on which $f$ acts as identity.

\section{Proposition 4.6.1.}

(1) For $a \in \mathbb{C}, H_{i}\left(\mathbb{C} f, \bar{M}_{\mathfrak{s l}_{2}}(a) \otimes \mathbb{C}_{\bar{\chi}_{-}}\right)= \begin{cases}\mathbb{C} & (i=0) \\ \{0\} & (i=1) .\end{cases}$

(2) For $a \in \mathbb{C}, H_{i}\left(\mathbb{C} f, \bar{L}_{\mathfrak{s l}_{2}}(a) \otimes \mathbb{C}_{\bar{\chi}_{-}}\right)= \begin{cases}\mathbb{C} & (i=0 \text { and } a \notin\{0,1,2, \ldots\}) \\ \{0\} & (\text { otherwise }) .\end{cases}$

(3) For $a \in \mathbb{C}, H_{i}\left(\mathbb{C} f, \bar{M}_{\mathfrak{s l}_{2}}(a)^{*} \otimes \mathbb{C}_{\bar{\chi}_{-}}\right)= \begin{cases}\mathbb{C} & (i=0) \\ \{0\} & (i=1) .\end{cases}$

(4) For any object $V$ in $\mathcal{O}\left(\mathfrak{s l}_{2}\right)$, we have $H_{1}\left(\mathbb{C} f, V \otimes \mathbb{C}_{\bar{\chi}_{-}}\right)=\{0\}$.

Proof. (1) Since $\bar{M}_{\mathfrak{s l}_{2}}(a)$ is free over $\mathbb{C} f$, so is $\bar{M}_{\mathfrak{s l}_{2}}(a) \otimes \mathbb{C}_{\bar{\chi}_{-}}$. (2) The case when $a \notin\{0,1,2, \ldots\}$ follows from (1). Otherwise $\bar{L}_{\mathfrak{s l}_{2}}(a)$ is finite-dimensional. Hence, $f$ acts nilpotently on $\bar{L}_{\mathfrak{s l}_{2}}(a)$. But then, the corresponding Chevalley complex is acyclic, by the argument of [7. Theorem 2.3]. (3) The case when $a \notin\{0,1,2, \ldots\}$ follows from (1). Otherwise we have the following exact sequence in $\mathcal{O}\left(\mathfrak{s l}_{2}\right)$ :

$$
0 \rightarrow \bar{L}_{\mathfrak{s l}_{2}}(a) \rightarrow \bar{M}_{\mathfrak{s l}_{2}}(a)^{*} \rightarrow \bar{M}_{\mathfrak{s l}_{2}}(-a-2) \rightarrow 0 .
$$


Hence, (3) follows from (1) and (2) by considering the associated long exact sequence of the Lie algebra homology. (4) In view of [1, Theorem 8.2], the above result (3) says that $H_{1}\left(\mathbb{C} f, I \otimes \mathbb{C}_{\bar{\chi}_{-}}\right)=\{0\}$ for any injective object $I$ in $\mathcal{O}\left(\mathfrak{s l}_{2}\right)$. For a given $V \in \operatorname{Obj} \mathcal{O}\left(\mathfrak{s l}_{2}\right)$, let $0 \rightarrow V \rightarrow I \rightarrow V / I \rightarrow 0$ be an exact sequence in $\mathcal{O}\left(\mathfrak{s l}_{2}\right)$ such that $I$ is injective. Then the associated long exact sequence proves $H_{1}\left(\mathbb{C} f, V \otimes \mathbb{C}_{\bar{\chi}_{-}}\right)=\{0\}$.

4.7 .

Proposition 4.7.1. For any $\lambda \in \widehat{\mathfrak{h}}^{*}$ we have

$$
\begin{aligned}
& \text { (1) } H^{i}(L(\lambda))_{\xi_{\lambda}}= \begin{cases}\mathbb{C} & \left(\text { if } i=0 \text { and }\left\langle\lambda, \alpha_{0}^{\vee}\right\rangle \notin\{0,1,2, \ldots\}\right), \\
\{0\} & (\text { otherwise }),\end{cases} \\
& \text { (2) } H^{i}\left(M(\lambda)^{*}\right)_{\xi_{\lambda}}= \begin{cases}\mathbb{C} & (\text { if } i=0), \\
\{0\} & (\text { otherwise }) .\end{cases}
\end{aligned}
$$

Proof. Observe that $L(\lambda)_{\xi_{\lambda}}$ is isomorphic to $\bar{L}_{\mathfrak{s l}_{2}}\left(\left\langle\lambda, \alpha_{0}^{\vee}\right\rangle\right)$ as a module over the subalgebra $\mathfrak{s l}_{2}$ generated by $e(-1)$ and $f(1)$. Similarly $M(\lambda)_{\xi_{\lambda}}^{*}$ is isomorphic to $\bar{M}_{\mathfrak{S I}_{2}}\left(\left\langle\lambda, \alpha_{0}^{\vee}\right\rangle\right)^{*}$. Hence, Proposition follows from Lemma 4.5.1 and Proposition 4.6 .1

4.8. Consider the Lie algebra homology $H_{\bullet}\left(\mathbb{C} e(-1), V_{\xi} \otimes \mathbb{C}_{\chi}\right), V \in \mathcal{O}_{k}$. On it there is a natural action of $\widehat{\mathfrak{t}}: H_{\bullet}\left(\mathbb{C} e(-1), V \otimes \mathbb{C}_{\chi}\right)=\bigoplus_{\xi \in \hat{\mathfrak{t}}^{*}} H_{\bullet}\left(\mathbb{C} e(-1), V \otimes \mathbb{C}_{\chi}\right)_{\xi}$, $H_{\bullet}\left(\mathbb{C} e(-1), V \otimes \mathbb{C}_{\chi}\right)_{\xi}=H_{\bullet}\left(\mathbb{C} e(-1), V_{\xi} \otimes \mathbb{C}_{\chi}\right)$.

Proposition 4.8.1. Let $V$ be any object of $\mathcal{O}_{k}$. Then,

(1) $H_{1}\left(\mathbb{C} e(-1), V \otimes \mathbb{C}_{\chi}\right)=\{0\}$.

(2) $H_{0}\left(\mathbb{C} e(-1), V \otimes \mathbb{C}_{\chi}\right)=\bigoplus_{\xi \leq \xi_{\lambda}} H_{0}\left(\mathbb{C} e(-1), V \otimes \mathbb{C}_{\chi}\right)_{\xi}$ and each weight space $H_{0}\left(\mathbb{C} e(-1), V \otimes \mathbb{C}_{\chi}\right)_{\xi}$ is finite-dimensional.

Proof. (1) It is sufficient to show that $H_{1}\left(\mathbb{C} e(-1), V_{\xi} \otimes \mathbb{C}_{\chi}\right)=\{0\}$ for all $V_{\xi}$. Viewed as a module over $\mathfrak{s l}_{2}$ generated by $e(-1)$ and $f(1)$, each $V_{\xi}$ can be obtained as an injective limit of objects of $\mathcal{O}\left(\mathfrak{s l}_{2}\right)$. Since the homology functor commutes with injective limits, Proposition follows from Proposition4.6.1 (4). (2) The first statement is obvious by (43). Thus, we have only to show that

$$
\operatorname{dim} H_{0}\left(\mathbb{C} e(-1), V \otimes \mathbb{C}_{\chi}\right)_{\xi}<\infty
$$

for each $\xi$. But it is easy to see that (45) holds for $V=M(\lambda), \lambda \in \widehat{\mathfrak{h}}^{*}$, and hence for any highest weight module. But the above result (1) says that the correspondence $V \rightsquigarrow H_{0}\left(\mathbb{C} e(-1), V \otimes \mathbb{C}_{\chi}\right)$ is exact. Therefore, one sees that 45olds for any $V$, by considering a highest weight filtration of $V$.

4.9. We end this section with the following important proposition.

Proposition 4.9.1. For any object $V$ in $\mathcal{O}_{k}$, each weight space $H^{\bullet}(V)_{\xi}, \xi \in \widehat{\mathfrak{t}}^{*}$, is finite-dimensional. Moreover, if $V \in \operatorname{Obj}_{\bar{k}}^{\leq \lambda}$, then, for any $i \in \mathbb{Z}, H^{i}(V)_{\xi}=\{0\}$ unless $\left\langle\xi_{\lambda}-\xi, \mathbf{D}^{\mathcal{W}}\right\rangle \geq \frac{1}{2}|i|$.

Proof. Decompose $\mathcal{F}\left(L \mathfrak{g}_{>0}\right)$ as $\mathcal{F}\left(L \mathfrak{g}_{>0}\right)=\mathcal{F}\left(L \mathfrak{g}_{>0} / \mathbb{C} e(-1)\right) \otimes \Lambda(\mathbb{C} e(-1))$, where $\mathcal{F}\left(L\left(\mathfrak{g}_{>0}\right) / \mathbb{C} e(-1)\right)$ is the subspace of $\mathcal{F}\left(L\left(\mathfrak{g}_{>0}\right)\right)$ spanned by the vectors

$$
\psi_{\alpha_{1}}\left(m_{1}\right) \ldots \psi_{\alpha_{r}}\left(m_{r}\right) \psi^{\beta_{1}}\left(n_{1}\right) \ldots \psi^{\beta_{s}}\left(n_{s}\right) \mathbf{1}
$$


with $\alpha_{i}, \beta_{i} \in \Delta_{>0}, m_{i} \leq\left\{\begin{array}{ll}-2 & \left(\text { if } \alpha_{i}=\theta\right) \\ -1 & \text { (otherwise), }\end{array} n_{i} \leq 0\right.$. Then,

$$
\mathcal{F}^{n}\left(L \mathfrak{g}_{>0}\right)=\sum_{i-j=n} \mathcal{F}^{i}\left(L \mathfrak{g}_{>0} / \mathbb{C} e(-1)\right) \otimes \Lambda^{j}(\mathbb{C} e(-1)),
$$

where $\mathcal{F}^{i}\left(L \mathfrak{g}_{>0} / \mathbb{C} e(-1)\right)=\mathcal{F}\left(L \mathfrak{g}_{>0} / \mathbb{C} e(-1)\right) \cap \mathcal{F}^{i}\left(L \mathfrak{g}_{>0}\right)$.

Set

$$
G^{p} C^{n}(V)=V \otimes \mathcal{F}^{\mathrm{ne}}(\chi) \otimes \sum_{\substack{i-j=n \\ i \geq p}} \mathcal{F}^{i}\left(L \mathfrak{g}_{>0} / \mathbb{C} e(-1)\right) \otimes \Lambda^{j}(\mathbb{C} e(-1)) \subset C^{n}(V) .
$$

Then,

$$
\begin{aligned}
& C^{n}(V)=G^{n} C^{n}(V) \supset G^{n+1} C^{n}(V) \supset G^{n+2} C^{n}(V)=\{0\}, \\
& d G^{p} C^{n}(V) \subset G^{p} C^{n+1}(V) .
\end{aligned}
$$

The corresponding the spectral sequence $E_{r} \Rightarrow H^{\bullet}(V)$ is the (semi-infinite and Lie superalgebra analogue of) Hochschild-Serre spectral sequence for the ideal $\mathbb{C} e(-1) \subset$ $L \mathfrak{g}_{>0}$ : By the definition,

$$
E_{1}^{p, q}=H_{-q}\left(\mathbb{C} e(-1), V \otimes \mathcal{F}^{\mathrm{ne}}(\chi)\right) \otimes \mathcal{F}^{p}\left(L \mathfrak{g}_{>0} / \mathbb{C} e(-1)\right),
$$

since the complex $\left(\sum_{p} G^{p} C(V) / G^{p+1} C(V), d\right)$ is nothing but the corresponding Chevalley complex. By Proposition 4.8.1 (1), we have

$$
E_{1}^{p, q} \cong \begin{cases}H_{0}\left(\mathbb{C} e(-1), V \otimes \mathbb{C}_{\chi}\right) \otimes \mathcal{F}^{\mathrm{ne}}(\chi) \otimes \mathcal{F}^{p}\left(L \mathfrak{g}_{>0} / \mathbb{C} e(-1)\right) & (q=0) \\ \{0\} & (q \neq 0)\end{cases}
$$

as $\hat{\mathfrak{t}}$-modules for any $p$.

Next observe that

$$
\begin{aligned}
& \mathcal{F}^{p}\left(L \mathfrak{g}_{>0} / \mathbb{C} e(-1)\right)=\bigoplus_{\xi \leq 0} \mathcal{F}^{p}\left(L \mathfrak{g}_{>0} / \mathbb{C} e(-1)\right)_{\xi}, \\
& \operatorname{dim}_{\mathbb{C}} \mathcal{F}^{p}\left(L \mathfrak{g}_{>0} / \mathbb{C} e(-1)\right)_{\xi}<\infty(\forall \xi) \\
& \mathcal{F}^{p}\left(L \mathfrak{g}_{>0} / \mathbb{C} e(-1)\right)_{\xi}=\{0\} \text { unless }\left\langle\xi, \mathbf{D}^{\mathcal{W}}\right\rangle \leq-\frac{1}{2}|p| .
\end{aligned}
$$

Hence, by (41), Proposition 4.8.1 (2) and (47), it follows that

$$
E_{1}^{p, 0}=\bigoplus_{\substack{\xi \leq \xi_{\lambda} \\\left\langle\xi_{\lambda}-\xi, \mathbf{D} \mathcal{W}\right\rangle \geq \frac{1}{2}|p|}}\left(E_{1}^{p, 0}\right)_{\xi}, \quad \operatorname{dim}_{\mathbb{C}}\left(E_{1}^{p, 0}\right)_{\xi}<\infty(\forall \xi)
$$

as $\widehat{\mathfrak{t}}$-modules. Proposition is proved since our filtration is compatible with the action of $\widehat{\mathfrak{t}}$.

\section{KaC-Roan-Wakimoto CONSTRUCtion II: the $\mathcal{W}$-ALGEbra CONSTRUCtion OF SUPERCONFORMAL ALGEBRAS}

5.1. Let $V_{k}(\mathfrak{g})=U(\widehat{\mathfrak{g}}) \otimes_{U(\mathfrak{g} \otimes \mathbb{C}[t] \oplus \mathbb{C} K \oplus \mathbb{C D})} \mathbb{C}_{k} \in O \operatorname{Obj} \mathcal{O}_{k}$ be the universal affine vertex algebra associated to $\mathfrak{g}$ at the given level $k \in \mathbb{C}$. Here, $\mathbb{C}_{k}$ is the one-dimensional representation of $\mathfrak{g} \otimes \mathbb{C}[t] \oplus \mathbb{C} K \oplus \mathbb{C D}$ on which $\mathfrak{g} \otimes \mathbb{C}[t] \oplus \mathbb{C D}$ acts trivially and $K$ acts as $k$ id. Then, the space

$$
C\left(V_{k}(\mathfrak{g})\right)=V_{k}(\mathfrak{g}) \otimes \mathcal{F}^{\mathrm{ne}}(\chi) \otimes \mathcal{F}\left(L \mathfrak{g}_{>0}\right)
$$


has a natural vertex algebra structure, see [10. Let $|0\rangle=(1 \otimes 1) \otimes \mathbf{1}_{\chi} \otimes \mathbf{1}$ be the canonical vector. Let $Y(v, z) \in$ End $C\left(V_{k}(\mathfrak{g})\right)\left[\left[z, z^{-1}\right]\right]$ be the field corresponding to $v \in C\left(V_{k}(\mathfrak{g})\right)$. By the definition,

$$
\begin{aligned}
& Y(v(-1)|0\rangle, z)=v(z)=\sum_{n \in \mathbb{Z}} v(n) z^{-n-1} \quad(v \in \mathfrak{g}), \\
& Y\left(\Phi_{\alpha}(-1)|0\rangle, z\right)=\Phi_{\alpha}(z)=\sum_{n \in \mathbb{Z}} \Phi_{\alpha}(n) z^{-n-1} \quad\left(\alpha \in \Delta_{\frac{1}{2}}\right), \\
& Y\left(\psi_{\alpha}(-1)|0\rangle, z\right)=\psi_{\alpha}(z)=\sum_{n \in \mathbb{Z}} \psi_{\alpha}(n) z^{-n-1} \quad\left(\alpha \in \Delta_{>0}\right), \\
& Y\left(\psi_{-\alpha}(0)|0\rangle, z\right)=\psi_{-\alpha}(z)=\sum_{n \in \mathbb{Z}} \psi_{-\alpha}(n) z^{-n} \quad\left(\alpha \in \Delta_{>0}\right) .
\end{aligned}
$$

We have:

$$
[d, Y(v, z)]=Y(d v, z) \quad \text { for all } v \in C\left(V_{k}(\mathfrak{g})\right)
$$

see [10].

\subsection{Define}

$$
\mathcal{W}_{k}(\mathfrak{g}, f) \underset{\text { def }}{=} H^{0}\left(V_{k}(\mathfrak{g})\right) .
$$

By (50), $Y$ descends to a map

$$
Y: \mathcal{W}_{k}(\mathfrak{g}, f) \rightarrow \operatorname{End} \mathcal{W}_{k}(\mathfrak{g}, f)\left[\left[z, z^{-1}\right]\right] .
$$

Hence, $\mathcal{W}_{k}(\mathfrak{g}, f)$ has a vertex algebra structure. The vertex algebra $\mathcal{W}_{k}(\mathfrak{g}, f)$ is called the $\mathcal{W}$-(super) algebra associated to the pair $(\mathfrak{g}, f)$ at level $k$. By the definition, the vertex algebra $\mathcal{W}_{k}(\mathfrak{g}, f)$ naturally acts on $H^{i}(V), V \in \mathcal{O}_{k}, i \in \mathbb{Z}$. Thus, we get the family of factors $V \rightsquigarrow H^{i}(V)$, depending on $i \in \mathbb{Z}$, from $\mathcal{O}_{k}$ to the category of $\mathcal{W}_{k}(\mathfrak{g}, f)$-modules.

Remark 5.2.1. When $\mathfrak{g}$ is a Lie algebra and $f$ is a regular nilpotent element of $\mathfrak{g}$, $\mathcal{W}_{k}(\mathfrak{g}, f)=\mathcal{W}_{k}(\mathfrak{g})$, the $\mathcal{W}$-algebra defined by B. Feigin and E. Frenkel [5].

5.3. The vertex algebra $\mathcal{W}_{k}(\mathfrak{g}, f)$ has a superconformal algebra structure provided that the level $k$ is non-critical, i.e, $k+h^{\vee} \neq 0$, see [10. Here $h^{\vee}$ is the dual Coxeter number of $\mathfrak{g}$. Let $L(z)=\sum_{n \in \mathbb{Z}} L(n) z^{-n-2}$ be the corresponding Virasoro field. The explicit form of $L(z)$ is given in [10]. When $f=f_{\theta}$, its central charge is given by

$$
c(k)=\frac{k \operatorname{sdim} \mathfrak{g}}{k+h^{\vee}}-6 k+h^{\vee}-4 .
$$

Let

$$
S(z)=\sum_{n \in \mathbb{Z}} S(n) z^{-n-2}=2\left(k+h^{\vee}\right) L(z) .
$$

Then, $S(z)$ is well-defined for any level $k$.

Remark 5.3.1. Let $\widehat{\Omega}$ be the universal Casimir operator ( 9 ) of $\widehat{\mathfrak{g}}$ acting on $V \in \mathcal{O}_{k}$. Then,

$$
S(0)+2\left(k+h^{\vee}\right) \mathbf{D}^{\mathcal{W}}=\widehat{\Omega}
$$

on $H^{\bullet}(V)$. 
5.4. Let

$$
J^{(v)}(z)=\sum_{n \in \mathbb{Z}} J^{(v)}(n) z^{-n-1}=v(z)+\sum_{\beta, \gamma \in \Delta_{>0}}(-1)^{p(\gamma)}\left(\left[v, u_{\beta}\right] \mid u_{-\gamma}\right): \psi_{\gamma}(z) \psi^{\beta}(z):,
$$

for $v \in \mathfrak{g}_{\leq 0}$.

Let $C_{k}(\mathfrak{g})$ be the subspace of $C\left(V_{k}(\mathfrak{g})\right)$ spanned by the vectors

$$
J^{\left(u_{1}\right)}\left(m_{1}\right) \ldots J^{\left(u_{p}\right)}\left(m_{p}\right) \Phi_{\alpha_{1}}\left(n_{1}\right) \ldots \Phi_{\alpha_{q}}\left(n_{q}\right) \psi^{\beta_{1}}\left(s_{1}\right) \ldots \ldots \psi^{\beta_{r}}\left(s_{r}\right)|0\rangle
$$

with $u_{i} \in \mathfrak{g}_{\leq 0}, \alpha_{i} \in \Delta_{\frac{1}{2}}, \beta_{i} \in \Delta_{>0}, m_{i}, n_{i}, s_{i} \in \mathbb{Z}$. It was shown in [1] that $C_{k}(\mathfrak{g})$ is a vertex subalgebra and a subcomplex of $C\left(V_{k}(\mathfrak{g})\right)$, and that

$$
\mathcal{W}_{k}(\mathfrak{g}, f)=H^{0}\left(C_{k}(\mathfrak{g}), d\right)
$$

as vertex algebras.

\subsection{Let}

$$
\widehat{\mathfrak{g}}^{f}=\mathfrak{g}^{f} \otimes \mathbb{C}\left[t, t^{-1}\right] \oplus \mathbb{C} 1
$$

be the affine Lie superalgebra of $\mathfrak{g}^{f}$ with respect to the 2 -cocycle $(,)^{\natural}$, defined by

$$
\left(u \otimes t^{m}, v \otimes t^{n}\right)^{\natural}= \begin{cases}m \delta_{m, n}\left(\left(k+h^{\vee}\right)(u \mid v)-\frac{1}{2} \operatorname{str}_{\mathfrak{g}_{0}}(\operatorname{ad} u)(\operatorname{ad} v)\right) & \left(\text { if } u, v \in \mathfrak{g}_{0}\right) \\ 0 & \text { (otherwise). }\end{cases}
$$

Let $V_{k}^{\natural}\left(\mathfrak{g}^{f}\right)$ be the corresponding universal vertex affine algebra:

$$
V_{k}^{\natural}\left(\mathfrak{g}^{f}\right)=U\left(\widehat{\mathfrak{g}}^{f}\right) \otimes_{U\left(\mathfrak{g}^{f} \otimes \mathbb{C}[t] \oplus \mathbb{C} 1\right)} \mathbb{C} .
$$

Then, the correspondence

$$
v \otimes t^{n} \rightarrow J^{(v)}(n) \quad\left(v \otimes t^{n} \in \widehat{\mathfrak{g}}^{f}\right)
$$

defines a $V_{k}^{\natural}\left(\mathfrak{g}^{f}\right)$-module structure on $C(V), V \in \mathcal{O}_{k}$. In particular, we have an embedding of vertex algebras

$$
V_{k}^{\natural}\left(\mathfrak{g}^{f}\right) \hookrightarrow C_{k}(\mathfrak{g}) \subset C\left(V_{k}(\mathfrak{g})\right) .
$$

Theorem 5.5.1 (V. Kac and M. Wakimoto 11, Theorem 4.1]). There exist a filtration $\left\{F^{p} W_{k}(\mathfrak{g}, f)\right\}$ of $\mathcal{W}_{k}(\mathfrak{g}, f)=H^{0}\left(C_{k}(\mathfrak{g}), d\right)$ such that $\mathcal{W}_{k}(\mathfrak{g}, f)=\bigcup_{p} F^{p} \mathcal{W}_{k}(\mathfrak{g}, f)$, $\bigcap_{p} F^{p} \mathcal{W}_{k}(\mathfrak{g}, f)=\{0\}, \widehat{\mathfrak{t}} \cdot F^{p} \mathcal{W}_{k}(\mathfrak{g}, f) \subset F^{p} \mathcal{W}_{k}(\mathfrak{g}, f)(\forall p), F^{p} \mathcal{W}_{k}(\mathfrak{g}, f) \cdot F^{q} \mathcal{W}_{k}(\mathfrak{g}, f) \subset$ $F^{p+q} \mathcal{W}_{k}(\mathfrak{g}, f)(\forall p, q)$, and the associated graded vertex algebra $\operatorname{gr}^{F} \mathcal{W}_{k}(\mathfrak{g}, f)$ is isomorphic to $V_{k}^{\natural}\left(\mathfrak{g}^{f}\right)$.

Remark 5.5.2. Actually stronger results was proved in 11]: it was shown that $H^{i}\left(V_{k}(\mathfrak{g})\right)=H^{i}\left(C_{k}(\mathfrak{g}), d\right)=\{0\}(i \neq 0)$. Further, the explicit form of $\mathcal{W}_{k}(\mathfrak{g}, f)$ was given provided that $f=f_{\theta}$. 
5.6. Let $W^{(v)}(n)$ be the cocycle corresponding to $v(n) \in \widehat{\mathfrak{g}}^{f}$ via Theorem 5.5.1] Then,

$$
W^{(v)}(n) H^{\bullet}(V)_{\xi} \subset H^{\bullet}(V)_{\xi+\eta} \quad\left(\text { if } v(n) \in\left(\widehat{\mathfrak{g}}^{f}\right)_{\eta}\right) .
$$

In particular, we have

$$
\left[\mathbf{D}^{\mathcal{W}}, W^{(v)}(n)\right]=(n-j) W^{(v)}(n) \quad\left(v \in \mathfrak{g}_{-j}^{f}\right) .
$$

Moreover,

$$
W^{(h)}(0), h \in \mathfrak{h}^{f}, \text { acts as }\langle\xi, h\rangle \text { id on } H^{\bullet}(V)_{\xi},
$$

see [10. Also, $W^{(f)}(n)$ coincides with $S(n-1)$ up to nonzero multiplication.

\section{The highest Weight MOdules}

We assume that $f=f_{\theta}$ and the condition (32) is satisfied for the rest of the paper.

6.1. Decompose $\widehat{\mathfrak{g}}^{f}$ as

$$
\widehat{\mathfrak{g}}^{f}=\left(\widehat{\mathfrak{g}}^{f}\right)_{-} \oplus\left(\widehat{\mathfrak{g}}^{f}\right)_{0} \oplus\left(\widehat{\mathfrak{g}}^{f}\right)_{+}
$$

where

$$
\begin{aligned}
& \left(\widehat{\mathfrak{g}}^{f}\right)_{+}=\mathfrak{g}_{-1} \otimes \mathbb{C}[t] t^{2} \oplus\left(\mathfrak{g}_{-\frac{1}{2}} \oplus \mathfrak{n}_{0,-} \oplus \mathfrak{h}^{f}\right) \otimes \mathbb{C}[t] t \oplus \mathfrak{n}_{0,+} \otimes \mathbb{C}[t], \\
& \left(\widehat{\mathfrak{g}}^{f}\right)_{0}=\mathfrak{h}^{f} \oplus \mathbb{C} 1 \oplus f \otimes \mathbb{C} t^{1}, \\
& \left(\widehat{\mathfrak{g}}^{f}\right)_{-}=\left(\mathfrak{g}_{-1} \oplus \mathfrak{g}_{-\frac{1}{2}} \oplus \mathfrak{n}_{0,-}\right) \otimes \mathbb{C}\left[t^{-1}\right] \oplus\left(\mathfrak{h}^{f} \oplus \mathfrak{n}_{0,+}\right) \otimes \mathbb{C}\left[t^{-1}\right] t^{-1} .
\end{aligned}
$$

6.2. Let

$$
\widehat{\mathfrak{h}}_{\mathcal{W}}^{*}=\left(\mathfrak{h}^{f}\right)^{*} \times \mathbb{C} .
$$

Recall the $\mathcal{W}_{k}(\mathfrak{g}, f)$-module $V$ is called a highest weight module with highest weight $\phi=(\bar{\phi}, s) \in \widehat{\mathfrak{h}}_{\mathcal{W}}^{*}$ if there exists a non-zero vector $v$ (called a highest weight vector) such that

$$
\begin{aligned}
& \mathcal{W}_{k}(\mathfrak{g}, f) v=V, \\
& W^{(u)}(n) v=0 \quad\left(\text { if } v \otimes t^{n} \in\left(\widehat{\mathfrak{g}}^{f}\right)_{+}\right), \\
& W^{(h)}(0) v=\bar{\phi}(h) v \quad\left(\text { if } h \in \mathfrak{h}^{f}\right), \\
& S(0) v=s v
\end{aligned}
$$

A highest weight $V$ with a highest vector $v$ is called a Verma module if the map

$$
\begin{array}{ccc}
U\left(\left(\widehat{\mathfrak{g}}^{f}\right)_{-}\right) & \rightarrow & M \\
\left(u_{1} \otimes t^{n_{1}}\right) \ldots\left(u_{r} \otimes t^{n_{r}}\right) & \mapsto & W^{\left(u_{1}\right)}\left(n_{1}\right) \ldots W^{\left(u_{r}\right)}\left(n_{r}\right) v
\end{array}
$$

is isomorphism of $\mathbb{C}$-vector spaces. Let $\mathbf{M}(\phi)$ denote the Verma module of highest weight $\phi \in \widehat{\mathfrak{h}}_{\mathcal{W}}^{*}$. Then, $\mathbf{M}(\phi)$ has a unique simple quotient, and we shall denote it by $\mathrm{L}(\phi)$. By the definition,

$$
\left\{\mathrm{E}(\phi) \mid \phi \in \widehat{\mathfrak{h}}_{\mathcal{W}}^{*}\right\}
$$

is the complete set of isomorphism classes of irreducible highest weight representations of $\mathcal{W}_{k}(\mathfrak{g}, f)$. 
6.3. For $\lambda \in \widehat{\mathfrak{h}}_{k}^{*}$, set

$$
\phi_{\lambda}=\left(\lambda_{\mid \mathfrak{h}^{f}},|\lambda+\rho|^{2}-|\rho|^{2}-2\left(k+h^{\vee}\right)\left\langle\lambda, \mathbf{D}^{\mathcal{W}}\right\rangle\right) \in \widehat{\mathfrak{h}}_{\mathcal{W}}^{*}
$$

(cf. Remark 5.3.1). Here, $\rho=\bar{\rho}+h^{\vee} \Lambda_{0}$, and $\bar{\rho}$ is the half of the difference of the sum of positive even roots and the sum of positive odd roots of $\mathfrak{g}$. Then, the correspondence $\widehat{\mathfrak{h}}_{k}^{*} \ni \lambda \mapsto \phi_{\lambda} \in \widehat{\mathfrak{h}}_{\mathcal{W}}^{*}$ is a surjection.

By [11, Theorem 6.3], we have

$$
H^{i}(M(\lambda)) \cong \begin{cases}\mathbf{M}\left(\phi_{\lambda}\right) & (i=0) \\ \{0\} & (i \neq 0) .\end{cases}
$$

for all $\lambda \in \widehat{\mathfrak{h}}^{*}$. Note that, this in particular implies

$$
H^{0}(M(\lambda)) \text { is generated by } H^{0}(M(\lambda))_{\xi_{\lambda}} \text { over } \mathcal{W}_{k}(\mathfrak{g}, f)
$$

since $H^{0}(M(\lambda))_{\xi_{\lambda}}$ is one-dimensional and spanned by its highest weight vector.

6.4. A consequence of (663) is the following theorem, which can be proved in the same manner as [1] Theorem 8.1].

Theorem 6.4.1. For any object $V$ in $\mathcal{O}_{k}^{\triangle}$, we have $H^{i}(V)=\{0\}(i \neq 0)$. In particular, we have $H^{i}(P)=\{0\}(i \neq 0)$ for any projective object $P$ in $\mathcal{O}_{k}^{\leq \lambda}$, $\lambda \in \widehat{\mathfrak{h}}_{k}^{*}$.

\section{The Computation of $H^{\bullet}\left(M(\lambda)^{*}\right)$}

In this section we compute $H^{\bullet}\left(M(\lambda)^{*}\right), \lambda \in \widehat{\mathfrak{h}}_{k}^{*}$, using a spectral sequence which we shall define in Subsection 7.11 Basically, it is the Hochschild-Serre spectral sequence for the subalgebra $\mathbb{C} e(-1) \oplus \mathfrak{g}_{>0} \otimes \mathbb{C}[t] \subset L \mathfrak{g}_{>0}$.

7.1. Just as in (14), let

$$
N\left(\chi_{-}\right)=U\left(L \mathfrak{g}_{<0}\right) / U\left(L \mathfrak{g}_{<0}\right) \operatorname{ker} \chi_{-},
$$

where $\operatorname{ker} \chi_{-} \subset U\left(L \mathfrak{g}_{\leq-1}\right)$ is the kernel of the character $\chi_{-}$defined by

$$
\chi_{-}(u(m))=(e(-1) \mid u(m)) \quad\left(u \in \mathfrak{g}_{\leq-1}, m \in \mathbb{Z}\right) .
$$

Let $\Phi_{u}(n), u \in \mathfrak{g}_{<0}, n \in \mathbb{Z}$, denote the image of $u(n) \in L \mathfrak{g}_{<0}$ in $N\left(\chi_{-}\right)$. As before, we set $\Phi_{-\alpha}(n)=\Phi_{u_{-\alpha}}(n)\left(\alpha \in \Delta_{\frac{1}{2}}, n \in \mathbb{Z}\right)$. Then, the correspondence $\Phi_{\alpha}(n) \mapsto$ $\Phi_{-\alpha}(-n)\left(\alpha \in \Delta_{\frac{1}{2}}, n \in \mathbb{Z}\right)$ defines an anti-algebra isomorphism $N(\chi) \cong N\left(\chi_{-}\right)$.

7.2. Let $\mathcal{F}^{\mathrm{ne}}\left(\chi_{-}\right)$be the irreducible representation of $N\left(\chi_{-}\right)$generated by a vector $\mathbf{1}_{\chi_{-}}$such that $\Phi_{-\alpha}(n) \mathbf{1}_{\chi_{-}}=0$ for $\alpha \in \Delta_{\frac{1}{2}}$ and $n \geq 1$. As before, we define a semisimple action of $\widehat{\mathfrak{h}}$ on $\mathcal{F}^{\text {ne }}\left(\chi_{-}\right)$by $h \mathbf{1}_{\chi_{-}}=0, \Phi_{-\alpha}(n) \mathcal{F}^{\text {ne }}\left(\chi_{-}\right)^{\lambda} \subset$ $\mathcal{F}^{\mathrm{ne}}\left(\chi_{-}\right)^{\lambda-\alpha+n \delta}\left(h \in \mathfrak{h}, \alpha \in \Delta_{\frac{1}{2}}, n \leq 0, \lambda \in \widehat{\mathfrak{h}}^{*}\right)$. Then, $\mathcal{F}^{\mathrm{ne}}\left(\chi_{-}\right)=\bigoplus_{\xi \in \widehat{\mathfrak{t}}^{*}} \mathcal{F}^{\mathrm{ne}}\left(\chi_{-}\right)_{\xi}$ and $\operatorname{dim} \mathcal{F}^{\text {ne }}\left(\chi_{-}\right)_{\xi}<\infty$ for all $\xi$. 
7.3. There exists a unique bilinear form

$$
\langle\cdot \mid \cdot\rangle^{\text {ne }}: \mathcal{F}^{\text {ne }}(\chi) \times \mathcal{F}^{\text {ne }}\left(\chi_{-}\right) \rightarrow \mathbb{C}
$$

such that $\left\langle\mathbf{1}_{\chi} \mid \mathbf{1}_{\chi_{-}}\right\rangle^{\mathrm{ne}}=1$ and $\left\langle\Phi_{\alpha}(m) v \mid v^{\prime}\right\rangle^{\text {ne }}=\left\langle v \mid \Phi_{-\alpha}(-m) v^{\prime}\right\rangle^{\text {ne }}\left(v \in \mathcal{F}^{\mathrm{ne}}(\chi)\right.$, $\left.v^{\prime} \in \mathcal{F}^{\text {ne }}\left(\chi_{-}\right), \alpha \in \Delta_{\frac{1}{2}}, m \in \mathbb{Z}\right)$. It is easy to see that this form is non-degenerate. Indeed, its restriction on $\mathcal{F}^{\text {ne }}(\chi)_{\xi} \times \mathcal{F}^{\text {ne }}\left(\chi_{-}\right)_{\xi}, \xi \in \widehat{\mathfrak{t}}^{*}$, is non-degenerate. Hence,

$$
\mathcal{F}^{\mathrm{ne}}(\chi)=\mathcal{F}^{\mathrm{ne}}\left(\chi_{-}\right)^{*}
$$

since each space $\mathcal{F}^{\text {ne }}\left(\chi_{-}\right)_{\xi}, \xi \in \widehat{\mathfrak{t}}^{*}$, decomposes into a finite sum of finite-dimensional weight spaces $\mathcal{F}^{\mathrm{ne}}\left(\chi_{-}\right)^{\lambda}$.

7.4. Let $\mathcal{C l}\left(L \mathfrak{g}_{<0}\right)$ be the Clifford superalgebra associated to $L \mathfrak{g}_{<0} \oplus\left(L \mathfrak{g}_{<0}\right)^{*}$ and its natural bilinear form. It is generated by the elements $\psi_{-\alpha}(n), \psi^{-\alpha}(n)\left(\alpha \in \Delta_{<0}\right.$, $n \in \mathbb{Z})$ with the relations $\left[\psi_{-\alpha}(m), \psi^{-\beta}(n)\right]=\delta_{\alpha, \beta} \delta_{m+n, 0}$. Here, the parity of $\psi_{-\alpha}(n)$ and $\psi^{-\alpha}(n)$ are reverse to $u_{-\alpha}$. We have an anti-algebra isomorphism $\mathcal{C l}\left(L \mathfrak{g}_{>0}\right) \cong \mathcal{C l}\left(L \mathfrak{g}_{<0}\right)$ defined by $\psi_{\alpha}(m) \mapsto(-1)^{p(\alpha)} \psi_{-\alpha}(-m), \psi^{\alpha}(m) \mapsto \psi^{-\alpha}(-m)$ $\left(\alpha \in \Delta_{>0}, m \in \mathbb{Z}\right)$.

7.5. Let $\mathcal{F}\left(L \mathfrak{g}_{<0}\right)$ be the irreducible representation of $\mathcal{C l}\left(L \mathfrak{g}_{<0}\right)$ generated by the vector $\mathbf{1}_{-}$such that $\psi_{-\alpha}(n) \mathbf{1}_{-}=0\left(\alpha \in \Delta_{>0}, n \geq 1\right), \psi^{-\alpha}(n) \mathbf{1}_{-}=0\left(\alpha \in \Delta_{>0}\right.$, $n \geq 0)$. As before, we have a natural action of $\widehat{\mathfrak{h}}$ on $\mathcal{F}\left(L \mathfrak{g}_{<0}\right)$.

There exists a unique bilinear form

$$
\langle\cdot \mid \cdot\rangle^{\mathrm{ch}}: \mathcal{F}\left(L \mathfrak{g}_{>0}\right) \times \mathcal{F}\left(L \mathfrak{g}_{<0}\right) \rightarrow \mathbb{C},
$$

which is non-degenerate on $\mathcal{F}\left(L \mathfrak{g}_{>0}\right)^{\lambda} \times \mathcal{F}\left(L \mathfrak{g}_{<0}\right)^{\lambda}, \lambda \in \widehat{\mathfrak{h}}^{*}$, such that $\left\langle\mathbf{1} \mid \mathbf{1}_{-}\right\rangle^{\text {ch }}=1$, $\left\langle\psi_{\alpha}(n) v \mid v^{\prime}\right\rangle^{\mathrm{ch}}=(-1)^{p(\alpha)}\left\langle v \mid \psi_{-\alpha}(-n) v^{\prime}\right\rangle^{\mathrm{ch}},\left\langle\psi^{\alpha}(n) v \mid v^{\prime}\right\rangle^{\mathrm{ch}}=\left\langle v \mid \psi^{-\alpha}(-n) v^{\prime}\right\rangle^{\mathrm{ch}}(v \in$ $\left.\mathcal{F}\left(L \mathfrak{g}_{>0}\right), v^{\prime} \in \mathcal{F}\left(L \mathfrak{g}_{<0}\right), \alpha \in \Delta_{>0}, n \in \mathbb{Z}\right)$. Hence,

$$
\mathcal{F}\left(L \mathfrak{g}_{>0}\right)=\mathcal{F}\left(L \mathfrak{g}_{<0}\right)^{*} .
$$

7.6. Let

$$
C_{-}(V)=V \otimes \mathcal{F}^{\mathrm{ne}}\left(\chi_{-}\right) \otimes \mathcal{F}\left(L \mathfrak{g}_{<0}\right) \quad\left(V \in \operatorname{Obj}_{k}\right) .
$$

Then, $C_{-}(V)=\bigoplus_{\lambda \in \widehat{\mathfrak{h}}^{*}} C_{-}(V)^{\lambda}$ with respect to the diagonal action of $\widehat{\mathfrak{h}}$. By (68) and (69), we have

$$
C\left(V^{*}\right)=C_{-}(V)^{*} \quad\left(V \in \operatorname{Obj}_{k}\right)
$$

as $\mathbb{C}$-vector spaces. Here, ${ }^{*}$ is defined by (11). Under the identification (71), we have

$$
(d g)(v)=g\left(d_{-} v\right) \quad\left(g \in C\left(V^{*}\right), v \in C(V)\right) .
$$

where

$$
\begin{aligned}
d_{-}=\sum_{\substack{\alpha \in \Delta_{>0} \\
n \in \mathbb{Z}}}(-1)^{p(\alpha)}\left(u_{-\alpha}(-n)+\Phi_{u_{-\alpha}}(-n)\right) \psi^{-\alpha}(n) \\
\quad-\frac{1}{2} \sum_{\substack{\alpha, \beta, \gamma \in \Delta_{>0} \\
k+l+m=0}}(-1)^{p(\alpha) p(\gamma)}\left(u_{\gamma} \mid\left[u_{-\alpha}, u_{-\beta}\right]\right) \psi^{-\alpha}(k) \psi^{-\beta}(l) \psi_{-\gamma}(m) .
\end{aligned}
$$


We have $d_{-}^{2}=0$. Also, $d_{-}$decomposes as

$$
\begin{aligned}
& d_{-}=d_{-}^{\chi}+d_{-}^{\text {st }}, \\
& \left(d_{-}^{\chi}\right)^{2}=\left(d_{-}^{\text {st }}\right)^{2}=\left\{d_{-}^{\chi}, d_{-}^{\text {st }}\right\}=0,
\end{aligned}
$$

where

$$
d_{-}^{\chi}=\sum_{\substack{\alpha \in \Delta_{1} \\ n \geq 1}}(-1)^{p(\alpha)} \Phi_{-\alpha}(n) \psi^{-\alpha}(-n)+\sum_{\alpha \in \Delta_{1}}(-1)^{p(\alpha)} \chi_{-}\left(u_{-\alpha}(1)\right) \psi^{-\alpha}(-1),
$$

and $d_{-}^{\text {st }}=d_{-}-d_{-}^{\chi}$.

Remark 7.6.1. The complex $\left(C_{-}(V), d_{-}\right)$is acyclic for any $V \in O b j \mathcal{O}_{k}$, since $f(1)$ acts locally nilpotently on $V$ (cf. [7, Theorem 2.3]).

7.7. The space $C_{-}\left(V_{k}(\mathfrak{g})\right)$ has a natural vertex algebra structure. The correspondences $v(n) \mapsto v^{t}(-n), \psi_{\alpha}(n) \mapsto(-1)^{p(\alpha)} \psi_{-\alpha}(-n), \psi^{\alpha}(n) \mapsto \psi^{-\alpha}(-n)$, $\Phi_{\alpha}(n) \mapsto \Phi_{-\alpha}(-n)$ extend to the anti-algebra homomorphism

$$
{ }^{t}: \mathcal{U}\left(C\left(V_{k}(\mathfrak{g})\right) \rightarrow \mathcal{U}\left(C_{-}\left(V_{k}(\mathfrak{g})\right)\right)\right.
$$

where $\mathcal{U}\left(C\left(V_{k}(\mathfrak{g})\right)\right)$ and $\mathcal{U}\left(C_{-}\left(V_{k}(\mathfrak{g})\right)\right)$ are universal enveloping algebras of $C\left(V_{k}(\mathfrak{g})\right)$ and $C_{-}\left(V_{k}(\mathfrak{g})\right)$ in the sense of [8] respectively. We have: $d_{-}=d^{t}, d_{-}^{\text {st }}=\left(d^{\text {st }}\right)^{t}$, $d_{-}^{\chi}=\left(d^{\chi}\right)^{t}$.

7.8. Let $J_{-}^{(v)}(n)=\left(J^{\left(v^{t}\right)}(-n)\right)^{t}$. Thus,

$J_{-}^{(v)}(z)=\sum_{n \in \mathbb{Z}} J_{-}^{(v)}(n) z^{-n-1}=v(z)+\sum_{\alpha, \beta \in \Delta_{>0}}(-1)^{p(\gamma)}\left(u_{\gamma} \mid\left[v, u_{-\beta}\right]\right): \psi_{-\gamma}(z) \psi^{-\beta}(z):$,

where $\psi_{-\alpha}(z)=\sum_{n \in \mathbb{Z}} \psi_{-\alpha}(n) z^{-n}, \psi^{-\alpha}(z)=\sum_{n \in \mathbb{Z}} \psi^{-\alpha}(z) z^{-n-1}\left(\alpha \in \Delta_{>0}\right)$.

Let $C_{-}(\lambda)$ be the subspace of $C_{-}(M(\lambda))$ spanned by the vectors

$$
J_{-}^{\left(u_{1}\right)}\left(m_{1}\right) \ldots J_{-}^{\left(u_{p}\right)}\left(m_{p}\right) \Phi_{-\alpha_{1}}\left(n_{1}\right) \ldots \Phi_{-\alpha_{q}}\left(n_{q}\right) \psi^{-\beta_{1}}\left(s_{1}\right) \ldots \ldots \psi^{-\beta_{r}}\left(s_{r}\right)|\lambda\rangle_{-}
$$

with $u_{i} \in \mathfrak{g}_{\geq 0}, \alpha_{i} \in \Delta_{\frac{1}{2}}, \beta_{i} \in \Delta_{>0}, m_{i}, n_{i}, s_{i} \in \mathbb{Z}$, where $|\lambda\rangle_{-}$is the canonical vector $v_{\lambda} \otimes \mathbf{1}_{\chi_{-}} \otimes \mathbf{1}_{-}$. Then, $d_{-} C_{-}(\lambda) \subset C_{-}(\lambda)$, i.e, $C_{-}(\lambda)$ is a subcomplex of $C_{-}(M(\lambda))$. Similarly, let $C_{-, k}(\mathfrak{g})$ be the subspace of $C_{-}\left(V_{k}(\mathfrak{g})\right)$ spanned by the elements

$$
J_{-}^{\left(u_{1}\right)}\left(m_{1}\right) \ldots J_{-}^{\left(u_{p}\right)}\left(m_{p}\right) \Phi_{-\alpha_{1}}\left(n_{1}\right) \ldots \Phi_{-\alpha_{q}}\left(n_{q}\right) \psi^{-\beta_{1}}\left(s_{1}\right) \ldots \ldots \psi^{-\beta_{r}}\left(s_{r}\right)|0\rangle_{-}
$$

with $u_{i} \in \mathfrak{g}_{\geq 0}, \alpha_{i} \in \Delta_{\frac{1}{2}}, \beta_{i} \in \Delta_{>0}, m_{i}, n_{i}, s_{i} \in \mathbb{Z}$, where $|0\rangle_{-}$is the canonical vector. Then, $d_{-} C_{-, k}(\mathfrak{g}) \subset C_{-, k}(\mathfrak{g})$. Also, $C_{-, k}(\mathfrak{g})$ is a vertex subalgebra of $C_{-}\left(V_{k}(\mathfrak{g})\right)$ and $C_{-}(\lambda), \lambda \in \widehat{\mathfrak{h}}^{*}$, is a module over $C_{-}\left(V_{k}(\mathfrak{g})\right)$.

Let the differential $d$ act on $C_{-}(\lambda)^{*}$ by $(d g)(v)=g\left(d_{-} v\right)\left(g \in C_{-}(\lambda)^{*}, v \in\right.$ $\left.C_{-}(\lambda)\right)$. The following proposition can be shown in the same manner as 11, Proposition 6.3].

Proposition 7.8.1. For any $\lambda \in \widehat{\mathfrak{h}}^{*}$, we have

$$
H^{\bullet}\left(M(\lambda)^{*}\right) \cong H^{\bullet}\left(C_{-}(\lambda)^{*}, d\right) .
$$

Remark 7.8.2. We have:

$$
H^{i}\left(C_{-}(\lambda)_{\xi}^{*}, d\right)=\operatorname{Hom}_{\mathbb{C}}\left(H^{-i}\left(\prod_{\substack{\mu \\ \xi_{\mu}=\xi}} C_{-}(\lambda)^{\mu}, d_{-}\right), \mathbb{C}\right) .
$$


7.9. The map (75) induces an anti-algebra homomorphism $\mathcal{U}\left(C_{k}(\mathfrak{g})\right) \rightarrow \mathcal{U}\left(C_{-, k}(\mathfrak{g})\right)$. This induces an anti-algebra homomorphism

$$
H^{0}\left(\mathcal{U}\left(C_{k}(\mathfrak{g})\right), \operatorname{ad} d\right) \rightarrow H^{0}\left(\mathcal{U}\left(C_{-, k}(\mathfrak{g})\right), \text { ad } d_{-}\right) .
$$

For $v(n) \in \widehat{\mathfrak{g}}^{f}$, let $W_{-}^{\left(v^{t}\right)}(-n)$ be the image of $W^{(v)}(n) \in H^{0}\left(\mathcal{U}\left(C_{k}(\mathfrak{g})\right)\right.$, ad $\left.d\right)$ under the map (76). Then,

$$
\left(W^{(u)}(n) f\right)(\widehat{v})=f\left(W^{\left(u^{t}\right)}(-n) \widehat{v}\right) \quad\left(u(n) \in \widehat{\mathfrak{g}}^{f}\right)
$$

for $f \in H^{\bullet}\left(C_{-}(\lambda)^{*}, d\right)$ and $\widehat{v} \in H^{\bullet}\left(\prod_{\xi_{\mu}=\xi} C_{-}(\lambda)^{\mu}, d_{-}\right)$, see Remark [7.8.2

7.10. Consider the weight space decomposition $C_{-}(\lambda)=\bigoplus_{\xi \in \hat{\mathfrak{t}}^{*}} C_{-}(\lambda)_{\xi}$ with respect to the action of $\widehat{\mathrm{t}}$. By Proposition 7.8.1 we have $H^{\bullet}\left(M(\lambda)^{*}\right)_{\xi}=H^{\bullet}\left(C_{-}(\lambda)_{\xi}^{*}, d\right)$. We also have $C_{-}(\lambda)_{\xi}=\{0\}$ unless $\xi \leq \xi_{\lambda}$.

Observe that the subcomplex $C_{-}(\lambda)_{\xi_{\lambda}} \subset C_{-}(\lambda)$ is spanned by the vectors

$$
J^{\left(e_{\theta}\right)}(-1)^{n}|\lambda\rangle_{-}, \quad J^{\left(e_{\theta}\right)}(-1)^{n} \psi^{-\theta}(-1)|\lambda\rangle_{-}
$$

with $n \in \mathbb{Z}_{\geq 0}$. Let

$$
G^{p} C_{-}(\lambda)_{\xi_{\lambda}}=\sum_{\substack{\mu \in \hat{\mathfrak{h}}^{*} \\\langle\mu-\lambda\rangle \geq-p}} C_{-}(\lambda)_{\xi_{\lambda}}^{\mu} \subset C_{-}(\lambda)_{\xi_{\lambda}} \quad(p \leq 0) .
$$

Then, $G^{p} C_{-}(\lambda)_{\xi_{\lambda}}$ is spanned by the vectors

$$
J^{\left(e_{\theta}\right)}(-1)^{n}|\lambda\rangle_{-}, \quad J^{\left(e_{\theta}\right)}(-1)^{n-1} \psi^{-\theta}(-1)|\lambda\rangle_{-} \quad(n \geq-p) .
$$

More generally, we define $G^{p} C_{-}(\lambda), p \leq 0$, as the subspace of $C_{-}(\lambda)$ spanned by the vectors

$$
J^{\left(u_{1}\right)}\left(m_{1}\right) \ldots J^{\left(u_{p}\right)}\left(m_{p}\right) \Phi_{-\alpha_{1}}\left(n_{1}\right) \ldots \Phi_{-\alpha_{q}}\left(n_{q}\right) \psi^{-\beta_{1}}\left(s_{1}\right) \ldots \ldots \psi^{-\beta_{r}}\left(s_{r}\right) v
$$

with $u_{i} \in \mathfrak{g}_{\geq 0}, \alpha_{i} \in \Delta_{\frac{1}{2}}, \beta_{i} \in \Delta_{>0}, m_{i}, n_{i}, s_{i} \in \mathbb{Z}, v \in G^{p} C_{-}(\lambda)_{\xi_{\lambda}}$. The $\widehat{\mathfrak{t}}$-weight space decomposition $G^{p} C_{-}(\lambda)=\bigoplus_{\substack{\xi \in \mathfrak{i}^{*} \\ \xi \leq \xi_{\lambda}}} G^{p} C_{-}(\lambda)_{\xi}$ agrees with (78).

7.11. We have:

$$
\begin{aligned}
& \cdots \subset G^{p} C_{-}(\lambda) \subset G^{p+1} C_{-}(\lambda) \subset \cdots \subset G^{0} C_{-}(\lambda)=C_{-}(\lambda), \\
& \bigcap_{p} G^{p} C_{-}(\lambda)=\{0\}, \\
& d_{-} G^{p} C_{-}(\lambda) \subset G^{p} C_{-}(\lambda) .
\end{aligned}
$$

Dually, let

$$
G^{p} C_{-}(\lambda)^{*}=\left(C_{-}(\lambda) / G^{p} C_{-}(\lambda)\right)^{*} \subset C_{-}(\lambda)^{*} \quad(p \leq 0) .
$$

Then,

$$
\begin{aligned}
& \cdots \supset G^{p} C_{-}(\lambda)^{*} \supset G^{p+1} C_{-}(\lambda)^{*} \supset \cdots \supset G^{0} C_{-}(\lambda)^{*}=\{0\}, \\
& C_{-}(\lambda)^{*}=\bigcup_{p} G^{p} C_{-}(\lambda)^{*} \\
& d G^{p} C_{-}(\lambda)^{*} \subset G^{p} C_{-}(\lambda)^{*} .
\end{aligned}
$$


Let $E_{r} \Rightarrow H^{\bullet}\left(C_{-}(\lambda)^{*}\right)=H^{\bullet}\left(M(\lambda)^{*}\right)$ be the corresponding spectral sequence. Since our filtration is compatible with the action of $\widehat{\mathfrak{t}}$, it is a direct sum of $\mathfrak{t}$-weight spaces:

$$
E_{r}=\bigoplus_{\xi \in \widehat{\mathfrak{t}}^{*}}\left(E_{r}\right)_{\xi}
$$

Below we compute this spectral sequence. Our goal is Theorem 7.17 .1

7.12. By the definition,

$$
E_{1}^{\bullet, q}=H^{q}\left(\mathrm{gr}^{G} C_{-}(\lambda)^{*}, d\right),
$$

where $\operatorname{gr}^{G} C_{-}(\lambda)^{*}=\sum_{p} G^{p} C_{-}(\lambda)^{*} / G^{p+1} C_{-}(\lambda)^{*}$. Note we have

$$
\operatorname{gr}^{G} C_{-}(\lambda)^{*}=\left(\operatorname{gr}^{G} C_{-}(\lambda)\right)^{*},
$$

where $\mathrm{gr}^{G} C_{-}(\lambda)=\sum_{p} G^{p} C_{-}(\lambda) / G^{p-1} C_{-}(\lambda)$.

7.13. Consider the subcomplex $\operatorname{gr}^{G} C_{-}(\lambda)_{\xi_{\lambda}} \subset \operatorname{gr}^{G} C_{-}(\lambda)$. By the definition, $\mathrm{gr}^{G} C_{-}(\lambda)_{\xi_{\lambda}}=\bigoplus_{p} G^{p} C_{-}(\lambda)_{\xi_{\lambda}} / G^{p-1} C_{-}(\lambda)_{\xi_{\lambda}}$ and $d_{-}^{\chi}$ acts trivially on $\mathrm{gr}^{G} C_{-}(\lambda)_{\xi_{\lambda}}$, see (78). Thus, $\left(\mathrm{gr}^{G} C_{-}(\lambda)_{\xi_{\lambda}}, d_{-}\right)=\left(C_{-}(\lambda)_{\xi_{\lambda}}, d_{-}^{\text {st }}\right)$. Hence,

$$
H^{\bullet}\left(\operatorname{gr}^{G} C_{-}(\lambda)_{\xi_{\lambda}}, d_{-}\right)=H^{\bullet}\left(C_{-}(\lambda)_{\xi_{\lambda}}, d_{-}^{\mathrm{st}}\right)=\bigoplus_{\mu \in \widehat{h}^{*}} H^{\bullet}\left(C_{-}(\lambda)_{\xi_{\lambda}}^{\mu}, d_{-}^{\mathrm{st}}\right) .
$$

Therefore,

$$
\left(E_{1}^{\bullet, q}\right)_{\xi_{\lambda}}=H^{q}\left(\mathrm{gr}^{G} C_{-}(\lambda)_{\xi_{\lambda}}^{*}, d^{\mathrm{st}}\right)=H^{q}\left(C_{-}(\lambda)_{\xi_{\lambda}}^{*}, d^{\mathrm{st}}\right)
$$

since each space $C_{-}(\lambda)_{\xi_{\lambda}}^{\mu}$ is finite-dimensional.

7.14. Each space $G^{p} C_{-}(\lambda)$ is a $C_{-, k}(\mathfrak{g})$-submodule of $C_{-}(\lambda)$. Thus, $\mathrm{gr}^{G} C_{-}(\lambda)$ has a $C_{-, k}(\mathfrak{g})$-module structure. We now describe this structure more precisely.

Define

$$
\overline{C_{-}(\lambda)}=C_{-}(\lambda) / G^{-1} C_{-}(\lambda) .
$$

Let $\overline{|\lambda\rangle}$ be the image of $|\lambda\rangle_{-}$in $\overline{C_{-}(\lambda)}$. Then,

$$
\begin{aligned}
& J_{-}^{(v)}(n) \overline{|\lambda\rangle}=0 \quad\left(v(n) \in L \mathfrak{g}_{\geq 0} \cap \widehat{\mathfrak{g}}_{+}\right), \\
& \psi^{-\alpha}(n) \overline{|\lambda\rangle}=0 \quad\left(\alpha \in \Delta_{>0}, n \geq 0\right), \\
& \Phi_{-\alpha}(n) \overline{|\lambda\rangle}=0 \quad\left(\alpha \in \Delta_{\frac{1}{2}}, n \geq 1\right), \\
& J^{(e)}(-1) \overline{|\lambda\rangle}=\psi^{-\theta}(-1) \overline{|\lambda\rangle}=0, \\
& J_{-}^{(h)}(0) \overline{|\lambda\rangle}=\langle\lambda, h\rangle \overline{|\lambda\rangle} \quad(h \in \mathfrak{h}) .
\end{aligned}
$$

We have the isomorphism of $C_{-, k}(\mathfrak{g})$-modules

$$
\bigoplus_{\mu \in \widehat{\mathfrak{h}}^{*}} \overline{C_{-}(\mu)} \otimes \operatorname{gr}^{G} C_{-}(\lambda)_{\xi_{\lambda}}^{\mu} \cong \operatorname{gr}^{G} C_{-}(\lambda),
$$

defined by

$$
\begin{aligned}
& J^{\left(u_{1}\right)}\left(m_{1}\right) \ldots J^{\left(u_{p}\right)}\left(m_{p}\right) \Phi_{-\alpha_{1}}\left(n_{1}\right) \ldots \Phi_{-\alpha_{q}}\left(n_{q}\right) \psi^{-\beta_{1}}\left(s_{1}\right) \ldots \ldots \psi^{-\beta_{r}}\left(s_{r}\right) \overline{|\mu\rangle} \otimes v \\
& \mapsto J^{\left(u_{1}\right)}\left(m_{1}\right) \ldots J^{\left(u_{p}\right)}\left(m_{p}\right) \Phi_{-\alpha_{1}}\left(n_{1}\right) \ldots \Phi_{-\alpha_{q}}\left(n_{q}\right) \psi^{-\beta_{1}}\left(s_{1}\right) \ldots \ldots \psi^{-\beta_{r}}\left(s_{r}\right) v
\end{aligned}
$$


$\left(u_{i} \in \mathfrak{g}_{\geq 0}, \alpha \in \Delta_{\frac{1}{2}}, \beta_{i} \in \Delta_{>0}, m_{i}, n_{i}, s_{i} \in \mathbb{Z}, v \in \operatorname{gr}^{G} C_{-}(\lambda)_{\xi_{\lambda}}^{\mu}\right)$. Here, the second factor $\mathrm{gr}^{G} C_{-}(\lambda)_{\xi_{\lambda}}^{\mu^{2}}$ is considered as a trivial $C_{-, k}(\mathfrak{g})$-module.

7.15. Let $d_{-}$act on $\overline{C_{-}(\mu)}$ by

$$
d_{-} b \overline{|\mu\rangle}=\left[d_{-}, b\right] \overline{|\mu\rangle} \quad\left(b \in \mathcal{U}\left(C_{-, k}(\mathfrak{g})\right)\right) .
$$

Under the identification (85), the action of $d_{-}$on $C_{-}(\lambda)$ is now described as follows:

$$
d_{-}(b \overline{|\mu\rangle} \otimes v)=\left[d_{-}, b\right] \overline{|\mu\rangle} \otimes v+(-1)^{p(b)} b \overline{|\mu\rangle} \otimes d_{-}^{\text {st }} v,
$$

for $b \in \mathcal{U}\left(C_{-, k}(\mathfrak{g})\right), v \in \operatorname{gr}^{G} C_{-}(\lambda)_{\xi_{\lambda}}$. Hence, we get the following lemma.

Lemma 7.15.1. We have:

$$
\operatorname{gr}^{G} C_{-}(\lambda)=\bigoplus_{\mu \in \widehat{\mathfrak{h}}^{*}} \overline{C_{-}(\mu)} \otimes C_{-}(\lambda)_{\xi_{\lambda}}^{\mu}
$$

as complexes, where the differential $d_{-}$acts on the right-hand-side by [86].

7.16. The following is easy to see.

Lemma 7.16.1. For each $\mu$, the complex $\left(\overline{C_{-}(\mu)}, d_{-}\right)$is a direct sum of finitedimensional subcomlexes $\overline{C_{-}(\mu)}$,$\xi \in \widehat{\mathfrak{t}}^{*}$.

Let

$$
\left(\widehat{\mathfrak{g}}^{e}\right)_{-}=\mathfrak{n}_{0,-} \otimes \mathbb{C}\left[t^{-1}\right] \oplus\left(\mathfrak{h}^{e} \oplus \mathfrak{n}_{0,+} \oplus \mathfrak{g}_{\frac{1}{2}}\right) \otimes \mathbb{C}\left[t^{-1}\right]^{-1} \oplus \mathfrak{g}_{1} \otimes \mathbb{C}\left[t^{-1}\right] t^{-2} .
$$

The following proposition can be shown in the same manner as [11, Theorem 4.1].

Proposition 7.16.2. Let $\mu \in \widehat{\mathfrak{h}}_{k}^{*}$.

(1) $H^{i}\left(\overline{C_{-}(\mu)}, d_{-}\right)=\{0\}(i \neq 0)$.

(2) The following map defines an isomorphism of $\mathbb{C}$-vector spaces.

$$
\begin{array}{ccc}
U\left(\left(\widehat{\mathfrak{g}}^{e}\right)_{-}\right) & \rightarrow & H^{0}\left(\overline{C_{-}(\mu)}, d_{-}\right) \\
u_{1}\left(n_{1}\right) \ldots u_{r}\left(n_{r}\right) & \mapsto & W_{-}^{\left(u_{1}\right)}\left(n_{1}\right) \ldots W_{-}^{\left(u_{r}\right)}\left(n_{r}\right) \overline{|\mu\rangle} .
\end{array}
$$

7.17. By Lemma 7.15.1 we have

$$
\operatorname{gr}^{G} C_{-}(\lambda)^{*}=\bigoplus_{\mu \in \widehat{\mathfrak{h}}^{*}}\left(\overline{C_{-}(\lambda)}\right)^{*} \otimes\left(C_{-}(\lambda)_{\xi_{\lambda}}^{*}\right)^{\mu}
$$

as complexes. Hence

$$
E_{1}^{\bullet, q}=\bigoplus_{\mu \in \widehat{\mathfrak{h}}^{*}} \bigoplus_{i+j=q} H^{i}\left(\left(\overline{C_{-}(\mu)}\right)^{*}, d\right) \otimes H^{j}\left(\left(C_{-}(\lambda)_{\xi_{\lambda}}^{*}\right)^{\mu}, d^{\mathrm{st}}\right)
$$

by (81) and the Künneth Theorem. But by Lemma [7.16.1] we have

$$
H^{i}\left(\left(\overline{C_{-}(\mu)}\right)^{*}, d\right)_{\xi}=\operatorname{Hom}_{\mathbb{C}}\left(H^{-i}\left(\overline{C_{-}(\mu)}, d_{-}\right)_{\xi}, \mathbb{C}\right)
$$

for each $i, \mu$ and $\xi$. Hence by Proposition $\overline{7.16 .2}(1)$, we have $H^{i}\left(\left(\overline{C_{-}(\mu)}\right)^{*}, d\right)=\{0\}$ $(i \neq 0)$. Thus,

$$
E_{1}^{\bullet, q}=\bigoplus_{\mu \in \widehat{\mathfrak{h}}^{*}} H^{0}\left(\left(\overline{C_{-}(\mu)}\right)^{*}, d\right) \otimes H^{q}\left(\left(C_{-}(\lambda)_{\xi_{\lambda}}^{*}\right)^{\mu}, d^{\mathrm{st}}\right) .
$$

But by Proposition 7.16.2 (2), we have

$$
H^{0}\left(\left(\overline{C_{-}(\mu)}\right)^{*}, d\right) \cong H^{0}\left(\left(\overline{C_{-}\left(\mu^{\prime}\right)}\right)^{*}, d\right)
$$


as $\mathbb{C}$-vector space for any $\mu, \mu^{\prime} \in \widehat{\mathfrak{h}}^{*}$. By (83), (90), and the identification (91), we get the isomorphism

$$
\begin{aligned}
E_{1}^{\bullet, q} & \cong H^{0}\left(\left(\overline{C_{-}(\lambda)}\right)^{*}, d\right) \otimes H^{q}\left(C_{-}(\lambda)_{\xi_{\lambda}}^{*}, d^{\mathrm{st}}\right) \\
& =H^{0}\left(\left(\overline{C_{-}(\lambda)}\right)^{*}, d\right) \otimes\left(E_{1}^{\bullet, q}\right)_{\xi_{\lambda}}
\end{aligned}
$$

of complexes, where the differential acts on the first factor $H^{0}\left(\left(\overline{C_{-}(\lambda)}\right)^{*}, d\right)$ trivially. This induces isomorphisms

$$
\left(E_{r}, d_{r}\right) \cong\left(H^{0}\left(\left(\overline{C_{-}(\lambda)}\right)^{*}, d\right) \otimes\left(E_{r}\right)_{\xi_{\lambda}}, 1 \otimes d_{r}\right)
$$

inductively for all $r \geq 1$. Therefore, we get

$$
E_{\infty} \cong H^{0}\left(\left(\overline{C_{-}(\lambda)}\right)^{*}, d\right) \otimes\left(E_{\infty}\right)_{\xi_{\lambda}} .
$$

Theorem 7.17.1. For any $\lambda \in \widehat{\mathfrak{h}}_{k}^{*}$, we have:

(1) $H^{i}\left(M(\lambda)^{*}\right)=\{0\}$ for all $i \neq 0$.

(2) $H^{0}\left(M(\lambda)^{*}\right)$ has a unique simple submodule $L$, and this $L$ satisfies $L_{\xi_{\lambda}} \neq$ $\{0\}$.

Proof. (1) Clearly, $\left(E_{r}\right)_{\xi_{\lambda}}$ converges to $H^{\bullet}\left(M(\lambda)^{*}\right)_{\xi_{\lambda}}$. Hence, by (94) and Proposition 4.7 .1 (2), it follows that

$$
H^{i}\left(M(\lambda)^{*}\right) \cong \begin{cases}H^{0}\left(\left(\overline{C_{-}(\lambda)}\right)^{*}, d\right) & (i=0) \\ \{0\} & (i \neq 0) .\end{cases}
$$

(2) Since the filtration $\left\{G^{p} C_{-}(\lambda)^{*}\right\}$ is compatible with the action of $C_{k}(\mathfrak{g})$, each $E_{r}$ and the resulting $\operatorname{gr}^{G} H^{\bullet}\left(M(\lambda)^{*}\right)=E_{\infty}$ are modules over $\mathcal{W}_{k}(\mathfrak{g}, f)$. Also, the isomorphism (92) compatible with the action of $W^{(n)}(v), v(n) \in\left(\widehat{\mathfrak{g}}^{f}\right)_{+}$. Hence, it follows that $\operatorname{gr}^{G} H^{0}\left(M(\lambda)^{*}\right) \cong H^{0}\left(\left(\overline{C_{-}(\lambda)}\right)^{*}, d\right)$ as a module over the subalgebra of $\mathcal{W}_{k}(\mathfrak{g}, f)$ generated by $W^{(u)}(n), u(n) \in\left(\widehat{\mathfrak{g}}^{f}\right)_{+}$. But then, by (89) and Proposition 7.16.2(2), it follows that

$$
\left\{v \in \operatorname{gr}^{G} H^{0}\left(M(\lambda)^{*}\right) \mid W^{(u)}(n) v=0\left(\forall u(n) \in\left(\widehat{\mathfrak{g}}^{f}\right)_{+}\right)\right\}=\operatorname{gr}^{G} H^{0}\left(M(\lambda)^{*}\right)_{\xi_{\lambda}},
$$

which is one-dimensional. Hence,

$$
\left\{v \in H^{0}\left(M(\lambda)^{*}\right) \mid W^{(u)}(n) v=0\left(\forall u(n) \in\left(\widehat{\mathfrak{g}}^{f}\right)_{+}\right)\right\}=H^{0}\left(M(\lambda)^{*}\right)_{\xi_{\lambda}} .
$$

Theorem is proved.

7.18. A consequence of Theorem 7.17.1 (1) is the following theorem, which can be proved in the same manner as [1, Theorem 8.1].

Theorem 7.18.1. For a given $\lambda \in \widehat{\mathfrak{h}}_{k}^{*}, H^{i}(I)=\{0\}(i \neq 0)$ for any injective object I in $\mathcal{O}_{k}^{\leq \lambda}$.

\section{Main Results}

8.1. V. Kac, S.-S. Roan and M. Wakimoto [10 conjectured the following.

Conjecture 1 (10, Conjecture $3.1 \mathrm{~B}]$ for $\mathcal{W}_{k}\left(\mathfrak{g}, f_{\theta}\right)$ ). If $L(\lambda)$ is an admissible $\widehat{\mathfrak{g}}-$ module, then the $\mathcal{W}_{k}\left(\mathfrak{g}, f_{\theta}\right)$-module $H^{\bullet}(L(\lambda))$ is either zero or irreducible. 
8.2. The following theorem is a consequence of Proposition 4.9.1 Theorem 6.4.1 and Theorem 7.18.1 which can be proved in the same manner as [1, Theorem 8.3].

Theorem 8.2.1. We have: $H^{i}(V)=\{0\}(i \neq 0)$ for any object $V$ in $\mathcal{O}_{k}$.

Corollary 8.2.2. The correspondence $V \rightsquigarrow H^{0}(V)$ defines an exact functor from $\mathcal{O}_{k}$ to the category of $\mathcal{W}_{k}(\mathfrak{g}, f)$-modules.

8.3 .

Theorem 8.3.1. We have

$$
H^{0}(L(\lambda)) \cong \begin{cases}L\left(\phi_{\lambda}\right) & \left(\text { if }\left\langle\lambda, \alpha_{0}^{\vee}\right\rangle \notin\{0,1,2, \ldots\}\right) \\ \{0\} & (\text { otherwise })\end{cases}
$$

for any $\lambda \in \widehat{\mathfrak{h}}_{k}^{*}$.

Proof. By [63), Theorem 7.17.1 (2) and Corollary 8.2.2 $H^{0}(L(\lambda))$ is either zero or isomorphic to $\mathrm{E}\left(\phi_{\lambda}\right)$. This can be proved in the same manner as [2, Theorem 6.5.1]. Also, by (64), $H^{0}(L(\lambda))=\{0\}$ if and only if $H^{0}(L(\lambda))_{\xi_{\lambda}}=\{0\}$. Hence Proposition 4.7 .1 (1) proves Theorem.

8.4. It is clear that Theorem 8.2.1 and Theorem8.3.1 prove Conjecture 1. Indeed, since the correspondence $\widehat{\mathfrak{h}}_{k}^{*} \ni \lambda \mapsto \phi_{\lambda} \in \widehat{\mathfrak{h}}_{\mathcal{W}}^{*}$ (see (62) ) is surjective, it follows that the character of any highest weight irreducible representation of $\mathcal{W}_{k}\left(\mathfrak{g}, f_{\theta}\right)$ at any level $k$ is determined by the character of the corresponding $\widehat{\mathfrak{g}}$-module $L(\lambda)$.

\section{REFERENCES}

[1] Arakawa, T.; Vanishing of Cohomology Associated to Quantized Drinfeld-Sokolov Reduction, Int. Math. Res. Notices, 2004, No. 15, 729-767.

[2] Arakawa, T.; Quantized Reductions and Irreducible Representations of $\mathcal{W}$-Algebras, preprint, math.QA/0403477

[3] Bershadsky, M.; Conformal field theory via Hamiltonian reduction, Comm. Math. Phys. 139 (1991) 71-82.

[4] Bershadsky, M.; Phys. Lett. 174B (1986) 285; Knizhnik, V.G.; Theor. Math. Phys., 66 (1986) 68.

[5] Feigin, B., Frenkel, E.: Quantization of Drinfeld-Sokolov Reduction, Phys. Lett. B 246, 75-81 (1990).

[6] Frenkel, E., Ben-Zvi, D.: Vertex algebras and algebraic curves, Mathematical Surveys and Monographs, 88.

[7] Frenkel, E., Kac, V., Wakimoto, M.: Characters and fusion rules for $W$-algebras via quantized Drinfeld-Sokolov reduction, Comm. Math. Phys. 147 (1992), no. 2, 295-328.

[8] Frenkel, I. B., Zhu, Y.: Vertex operator algebras associated to representations of affine and Virasoro algebras. Duke Math. J. 66 (1992), no. 1, 123-168.

[9] Kac, Victor G.: Infinite-dimensional Lie algebras, Cambridge University Press, 1990.

[10] Kac, V. G., Roan, S.-S., Wakimoto, M.: Quantum Reduction for Affine Superalgebras, Commun. Math. Phys. 241 (2003), 307-342.

[11] Kac, V. G., Wakimoto, M.: Quantum Reduction amd Representation Theory of Superconformal Algebras, preprint, math-ph/0304011 to appear in Adv. in Math.

Graduate school of Mathematics, Nagoya University, Chikusa-Ku, Nagoya, 464-8602, JAPAN

E-mail address: tarakawa@math.nagoya-u.ac.jp 